\title{
The SPHERE infrared survey for exoplanets (SHINE)
}

\section{Observations, data reduction and analysis, detection performances, and initial results ${ }^{\star}$}

\author{
M. Langlois ${ }^{1,2}$, R. Gratton ${ }^{3}$, A.-M. Lagrange ${ }^{4,5}$, P. Delorme ${ }^{4}$, A. Boccaletti ${ }^{5}$, M. Bonnefoy ${ }^{4}$, A.-L. Maire ${ }^{7,6}$,

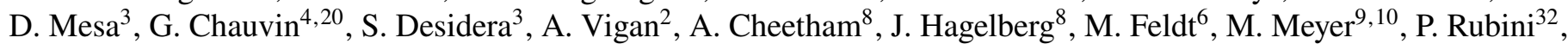 \\ H. Le Coroller ${ }^{2}$, F. Cantalloube ${ }^{6}$, B. Biller ${ }^{6,12,13}$, M. Bonavita ${ }^{11}$, T. Bhowmik ${ }^{5}$, W. Brandner ${ }^{6}$, S. Daemgen ${ }^{10}$, \\ V. D’Orazi ${ }^{3}$, O. Flasseur ${ }^{1}$, C. Fontanive ${ }^{13,3}$, R. Galicher ${ }^{5}$, J. Girard ${ }^{4}$, P. Janin-Potiron ${ }^{5}$, M. Janson ${ }^{28,6}$, M. Keppler ${ }^{6}$, \\ T. Kopytova ${ }^{6,30,29}$, E. Lagadec ${ }^{14}$, J. Lannier ${ }^{4}$, C. Lazzoni ${ }^{27}$, R. Ligi ${ }^{14}$, N. Meunier ${ }^{4}$, A. Perreti ${ }^{8}$, C. Perrot ${ }^{5,24,25}$, \\ L. $\operatorname{Rodet}^{4}$, C. Romero ${ }^{4,16}$, D. Rouan ${ }^{5}$, M. Samland ${ }^{28,6}$, G. Salter ${ }^{2}$, E. Sissa ${ }^{3}$, T. Schmidt ${ }^{5}$, A. Zurlo ${ }^{17,18,2}$, D. Mouillet ${ }^{4}$, \\ L. Denis ${ }^{26}$, E. Thiébaut ${ }^{1}$, J. Milli ${ }^{4}$, Z. Wahhaj ${ }^{16}$, J.-L. Beuzit ${ }^{2}$, C. Dominik ${ }^{19}$, Th. Henning ${ }^{6}$, F. Ménard ${ }^{4}$, A. Müller ${ }^{6}$, \\ H. M. Schmid ${ }^{10}$, M. Turatto ${ }^{3}$, S. Udry ${ }^{8}$, L. Abe ${ }^{14}$, J. Antichi ${ }^{3}$, F. Allard ${ }^{1}$, A. Baruffolo ${ }^{3}$, P. Baudoz ${ }^{5}$, J. Baudrand ${ }^{5}$, \\ A. Bazzon ${ }^{10}$, P. Blanchard ${ }^{2}$, M. Carbillet ${ }^{14}$, M. Carle ${ }^{2}$, E. Cascone ${ }^{3}$, J. Charton ${ }^{4}$, R. Claudi $^{3}$, A. Costille ${ }^{2}$, \\ V. De Caprio ${ }^{23}$, A. Delboulbé ${ }^{4}$, K. Dohlen ${ }^{2}$, D. Fantinel ${ }^{3}$, P. Feautrier ${ }^{4}$, T. Fusco ${ }^{21,2}$, P. Gigan ${ }^{5}$, E. Giro ${ }^{3}$, D. Gisler ${ }^{10}$, \\ L. Gluck ${ }^{4}$, C. Gry ${ }^{2}$, N. Hubin ${ }^{15}$, E. Hugot ${ }^{2}$, M. Jaquet ${ }^{2}$, M. Kasper ${ }^{15,4}$, D. Le Mignant ${ }^{2}$, M. Llored ${ }^{2}$, F. Madec ${ }^{2}$, \\ Y. Magnard ${ }^{4}$, P. Martinez ${ }^{14}$, D. Maurel ${ }^{4}$, S. Messina ${ }^{31}$, O. Möller-Nilsson ${ }^{21}$, L. Mugnier ${ }^{21}$, T. Moulin ${ }^{4}$, A. Origné2, \\ A. Pavlov ${ }^{6}$, D. Perret ${ }^{5}$, C. Petit ${ }^{21}$, J. Pragt ${ }^{4}$, P. Puget ${ }^{4}$, P. Rabou $^{4}$, J. Ramos ${ }^{4}$, F. Rigal ${ }^{4}$, S. Rochat ${ }^{4}$, R. Roelfsema ${ }^{22}$, \\ G. Rousset ${ }^{5}$, A. Roux ${ }^{4}$, B. Salasnich ${ }^{3}$, J.-F. Sauvage ${ }^{21,2}$, A. Sevin ${ }^{5}$, C. Soenke ${ }^{15}$, E. Stadler ${ }^{4}$, \\ M. Suarez ${ }^{15}$, L. Weber ${ }^{8}$, F. Wildi ${ }^{8}$, and E. Rickman ${ }^{8}$
}

(Affiliations can be found after the references)

Received 24 October 2020 / Accepted 6 February 2021

\begin{abstract}
Context. In recent decades, direct imaging has confirmed the existence of substellar companions (exoplanets or brown dwarfs) on wide orbits $(>10 \mathrm{au})$ around their host stars. In striving to understand their formation and evolution mechanisms, in 2015 we initiated the SPHERE infrared survey for exoplanets (SHINE), a systematic direct imaging survey of young, nearby stars that is targeted at exploring their demographics.

Aims. We aim to detect and characterize the population of giant planets and brown dwarfs beyond the snow line around young, nearby stars. Combined with the survey completeness, our observations offer the opportunity to constrain the statistical properties (occurrence, mass and orbital distributions, dependency on the stellar mass) of these young giant planets.

Methods. In this study, we present the observing and data analysis strategy, the ranking process of the detected candidates, and the survey performances for a subsample of 150 stars that are representative of the full SHINE sample. Observations were conducted in a homogeneous way between February 2015 and February 2017 with the dedicated ground-based VLT/SPHERE instrument equipped with the IFS integral field spectrograph and the IRDIS dual-band imager, covering a spectral range between 0.9 and $2.3 \mu \mathrm{m}$. We used coronographic, angular, and spectral differential imaging techniques to achieve the best detection performances for this study, down to the planetary mass regime.

Results. We processed, in a uniform manner, more than 300 SHINE observations and datasets to assess the survey typical sensitivity as a function of the host star and of the observing conditions. The median detection performance reached $5 \sigma$-contrasts of $13 \mathrm{mag}$ at 200 mas and $14.2 \mathrm{mag}$ at 800 mas with the IFS ( $Y J$ and $Y J H$ bands), and of $11.8 \mathrm{mag}$ at $200 \mathrm{mas}, 13.1 \mathrm{mag}$ at $800 \mathrm{mas}$, and $15.8 \mathrm{mag}$ at 3 as with IRDIS in $H$ band, delivering one of the deepest sensitivity surveys thus far for young, nearby stars. A total of sixteen substellar companions were imaged in this first part of SHINE: seven brown dwarf companions and ten planetary-mass companions. These include two new discoveries, HIP 65426 b and HIP 64892 B, but not the planets around PDS70 that had not been originally selected for the SHINE core sample. A total of 1483 candidates were detected, mainly in the large field of view that characterizes IRDIS. The color-magnitude diagrams, low-resolution spectrum (when available with IFS), and follow-up observations enabled us to identify the nature (background contaminant or comoving companion) of about $86 \%$ of our subsample. The remaining cases are often connected to crowded-field follow-up observations that were missing. Finally, even though SHINE was not initially designed for disk searches, we imaged twelve circumstellar disks, including three new detections around the HIP 73145, HIP 86598, and HD 106906 systems.
\end{abstract}

\footnotetext{
${ }^{\star}$ Full Table A.1 is only available at the CDS via anonymous ftp to cdsarc.u-strasbg.fr (130.79.128.5) or via http://cdsarc. u-strasbg.fr/viz-bin/cat/J/A+A/651/A71
} 
A\&A 651, A71 (2021)

\begin{abstract}
Conclusions. Nowadays, direct imaging provides a unique opportunity to probe the outer part of exoplanetary systems beyond 10 au to explore planetary architectures, as highlighted by the discoveries of: one new exoplanet, one new brown dwarf companion, and three new debris disks during this early phase of SHINE. It also offers the opportunity to explore and revisit the physical and orbital properties of these young, giant planets and brown dwarf companions (relative position, photometry, and low-resolution spectrum in near-infrared, predicted masses, and contrast in order to search for additional companions). Finally, these results highlight the importance of finalizing the SHINE systematic observation of about 500 young, nearby stars for a full exploration of their outer part to explore the demographics of young giant planets beyond $10 \mathrm{au}$ and to identify the most interesting systems for the next generation of high-contrast imagers on very large and extremely large telescopes.
\end{abstract}

Key words. methods: observational - planets and satellites: detection - techniques: image processing - methods: statistical instrumentation: high angular resolution - planets and satellites: formation

\section{Introduction and context}

The discovery of the first brown dwarf companion G1229 B benefited from the combined technological innovation of infrared detectors and high contrast techniques (Nakajima et al. 1995). Following this discovery, the first generation of dedicated planet imagers on 10-m class telescopes ( $\mathrm{NaCo}$ at VLT, NIRC2 at Keck, NICI at Gemini) conducted systematic surveys of young and nearby stars. These led the first direct detections of planetary mass companions in the early 2000s. These companions were detected at distances larger than several hundreds of astronomical units (au) or with a mass ratio not much smaller than a tenth with respect to their host primaries (except for brown dwarf primaries), giving hints at a formation scenario taking place via gravo-turbulent fragmentation (Hennebelle \& Chabrier 2011) or gravitational disk instability (Boss 1997). Thanks to the improvement of direct imaging observation and data analysis techniques with ground-based adaptive optics systems (AO) or space telescopes, a few planetary-mass objects and low-mass brown dwarfs have been detected since the first detection by Chauvin et al. (2004). Moreover, these developments, enabled the discoveries of giant planets within 100 au around such young, nearby, and dusty early-type stars as HR 8799 b,c,d,e (Marois et al. 2008, 2010), $\beta$ Pictoris b (Lagrange et al. 2009), and more recently HD 95086 b (Rameau et al. 2013a), and GJ 504 b (Kuzuhara et al. 2013).

Direct imaging is the only viable technique for carrying out probes for planets at large separations with single epoch observations; however, such detections require us to overcome the difficulties caused by the angular proximity and the high contrast involved. With improved instruments and data reduction techniques, we are currently initiating a characterization of the giant planet population at wide orbits, typically between 10 and $100 \mathrm{au}$. More than a decade of direct imaging surveys targeting several hundred young, nearby stars have lead to the discovery of approximately a dozen sub-stellar companions, excluding the companions to brown dwarfs located in the star vicinity (within $100 \mathrm{au}$ ). Despite the relatively small number of discoveries compared with other techniques, such as radial velocity and transit, each new imaged giant planet has provided unique clues on the formation, evolution and physics of young massive Jupiters.

Early surveys performed using the first generation of planet imagers made it possible to conduct systematic surveys that are relatively modest in their size, each sampling less than a few hundreds of young nearby stars (Chauvin et al. 2018). Various strategies were followed for the target selection of these surveys: (i) complete census of given associations (Chauvin et al. 2003, 2010; Masciadri et al. 2005; Kasper et al. 2007); (ii) selection of young, intermediate-mass stars (Janson et al. 2011; Rameau et al. 2013b; Nielsen et al. 2013); (iii) or very low-mass stars (Delorme et al. 2012; Chauvin et al. 2012; Bowler et al. 2015;
Lannier et al. 2016); (iv) application of figures of merit considering detection rate with toy models of planet populations (Biller et al. 2013). Numerous direct imaging surveys for detecting giant planet companions have reported null detections (Masciadri et al. 2005; Kasper et al. 2007; Chauvin et al. 2010; Biller et al. 2007; Lafrenière et al. 2007; Ehrenreich et al. 2010; Janson et al. 2011; Delorme et al. 2012), but this allowed us to set upper limits to the occurrence of giant planets.

Ongoing surveys target several hundreds of stars, with the largest surveys to date due to be completed in the next years. The SpHere INfrared survey for Exoplanets (SHINE, Chauvin et al. 2017b), Gemini GPIES (Macintosh et al. 2015), and SCExAO (Jovanovic et al. 2016) now combine dedicated extreme adaptive optics systems with coronagraphic and both angular and spectral differential image processing. With enhanced detection performances, the objective is to significantly increase the number of imaged planets to characterize, but also to provide better statistical constraints on the occurrence and the characteristics of exoplanets at wide orbits (>10 au). This should give us a more global picture of planetary systems architecture at all orbits to improve our understanding of planetary formation and evolution mechanisms. Early results have already confirmed the gain in terms of detection performance compared with the first generation of planet imagers (Nielsen et al. 2019; Vigan et al. 2021).

The near-infrared wavelengths ( $H$ and $K$-bands) have been used intensively. They are a good compromise between lowbackground noise, high angular resolution, and good Strehl correction. However, thermal imaging at the $L$ - or $M$-bands has been very competitive in terms of detection performances as the planet-star contrast and the Strehl correction are more favorable in those wavelengths, despite an increased thermal background and larger inner working angle. For instance, SPHERE could, in some cases, prove less sensitive than $\mathrm{NaCo}$ for the detection of giant planets around young, nearby $\mathrm{M}$ dwarfs at typical separations larger than 500-1000 mas.

Both SHINE and GPIES surveys recently discovered three new exoplanets (Macintosh et al. 2015; Chauvin et al. 2017b; Keppler et al. 2018) and a few additional higher-mass brown dwarfs (Konopacky et al. 2016; Cheetham et al. 2018). Smaller surveys using SPHERE and GPI also discovered several substellar companions (Milli et al. 2017a; Wagner et al. 2020; Bohn et al. 2020). These surveys offer unprecedented detection, astrometric, and spectrophotometric capabilities that allow us to characterize fainter and closer giant planets, such as the recent discovery of 51 Eri b ( $2 M_{\text {Jup }}$ at 14 au, T5-type, of an age of $20 \mathrm{Myr}$; Macintosh et al. 2015; Samland et al. 2017), HIP 65426 b, a young, warm, and dusty L5-L7 massive Jovian planet located at about 92 au from its host star (Chauvin et al. 2017b), and the young solar analogue PDS 70, which is now known to actually host two planets PDS $70 \mathrm{~b}$ discovered during the SHINE 
campaign (Keppler et al. 2018; Müller et al. 2018) and PDS 70 c by MUSE (Haffert et al. 2020). Such surveys also provide key spectral and orbital characterisation data for known exoplanets (e.g., De Rosa et al. 2016; Samland et al. 2017; Chauvin et al. 2018; Wang et al. 2018; Cheetham et al. 2019; Lagrange et al. 2019; Maire et al. 2019). Despite these new discoveries, SHINE and GPIES have yielded significantly fewer exoplanet detections than predicted, with their use of extrapolations of radial velocity planet populations to larger semi-major axes (e.g., Cumming et al. 2008). This results in our setting strong statistical constraints on the distribution of giant exoplanets at separations of $>10$ au from their stars, as well as sub-stellar companions to young stars (Nielsen et al. 2019; Vigan et al. 2021). As these systems are young $(<100 \mathrm{Myr})$, and thus closer to their epoch of formation than, for instance, radial-velocity planets (typically 1-10 Gyr), statistical analyses of large direct-imaging surveys can provide hints for the potential formation mechanism responsible for producing giant exoplanets on wide orbits.

This paper is part of a series of three papers aimed at describing early results obtained from an analysis of a subset of the SHINE data. This paper describes the observations, data quality, and analysis methods, presenting the results in terms of detections and upper limits for this subset of the SHINE data. Two associated papers describe the general characteristics of the survey sample (Desidera et al. 2021) and the statistical analysis, as well as a discussion of their implications for formation scenarios (Vigan et al. 2021).

Following the introduction, we present in Sect. 2 the observations that were performed to underpin this work. In Sect. 3, we described the reduction and calibration of the data sets. Section 4 is dedicated to the main results of the survey, including the detection limits of the survey. Finally, we summarize in Sect. 5 the characterization of newly discovered and known substellar companions, along with a description of the circumstellar disks detected among the survey data. The conclusions and prospects are drawn in Sect. 7. The relative astrometry and photometry of companion candidates from the sample are listed in Appendix A.

\section{SHINE survey}

The SpHere INfrared survey for Exoplanets (SHINE: Chauvin et al. 2017a) was designed for 200 telescope nights, allocated in visitor mode, using the SPHERE consortium guaranteed-time. It has been designed to: (i) identify and characterized new planetary and brown dwarf companions; (ii) study the architecture of planetary systems (multiplicity and dynamical interactions); (iii) investigate the link between the presence of planets and disks (in synergy with the GTO program aimed at disk characterization); (iv) determine the frequency of giant planets beyond $10 \mathrm{au}$; and (v) investigate the impact of stellar mass (and even age if possible) on the frequency and characteristics of planetary companions over the range $0.5-3.0 M_{\odot}$.

The SHINE survey started in February 2015 and will be completed in mid-2021, with observations of 500 targets out of a larger sample of 800 nearby young stars aimed at searching for new sub-stellar companions. The sample is oversized with respect to the available telescope time by a factor of approximately two, on the basis of the adopted observing strategy, which relies on observations surrounding meridian passage in order to achieve the maximum field-of-view (FoV) rotation for optimal angular differential imaging. This requires some flexibility in the target list in order to optimize the scheduling.
The general design of the survey, the sample selection, and the simulations performed for building it, as well as the parameters of the individual targets used in this early statistical analysis and the general properties of the sample used in this series of papers are described in detail in Desidera et al. (2021). In this paper, we describe the initial results obtained from the analysis of about a third of the large SHINE survey sample, considering only those targets whose first observations were carried out prior to Feb. 2017. Several second epochs observations were also performed between 2016 and 2019 to discriminate companions from background stars on the selected sample described in this paper. This selected sample dataset, which includes 150 targets, is already large enough for reviewing the survey efficiency, to discuss the incidence of massive planets at a separation of $>10$ au and to compile new indications regarding the formation scenarios for giant planets.

\subsection{Observation setup}

All the observations were performed with the SpectroPolarimeter High-contrast Exoplanet REsearch (SPHERE; Beuzit et al. 2019), combining its SAXO extreme adaptive optics system (Fusco et al. 2006, 2014; Sauvage et al. 2016) and its apodized pupil Lyot coronagraphs (Boccaletti et al. 2008; Carbillet et al. 2011; Guerri et al. 2011). Observations were acquired in either IRDIFS or IRDIFS-EXT mode, that is, with both NIR sub-systems, IFS (Claudi et al. 2008), and IRDIS (Dohlen et al. 2008) that were all carrying out observations in parallel (Zurlo et al. 2014; Mesa et al. 2015). The IFS covers a $1.7^{\prime \prime} \times 1.7^{\prime \prime}$ FoV and IRDIS covers a more or less circular, unvignetted FoV of diameter $\sim 9 "$. The APLC_YJHs and APLC_Ks coronagraphic configurations were used for the IRDIFS and IRDIFS-EXT observations, respectively, and in IRDIS, all first epoch observations were performed with the DB-H23 and DBK12 dual-band filter pairs (Vigan et al. 2010). Due to the presence of known companions or the detection of (new) candidate companions, some targets were observed multiple times for astrometric monitoring. In addition to the initial selected filters, follow-up observations were performed in different configurations with IRDIS, for example with the broad-band BB-H and dual-band DB-J23 filters, as listed in the filter column of Table 1. This resulted in a varying number of observations for each target.

\subsection{Optimized observation planning: SPOT}

Given the large number of targets, each associated with a certain level of priority and urgency (how soon the observation has to be made), along with the various observing constraints, including those connected to angular differential imaging (ADI: Marois et al. 2006) observations, we built a dedicated tool, named SPOT, to deliver an optimised scheduling of the observations, both on long and short terms and based on simulated annealing. It is described in in detail in Lagrange et al. (2016).

In brief, SPOT uses as its input the calendar of allocated observing nights, the list of targets available for (for instance) the whole semester, together with their associated instrumental setup (that is associated with specific overheads) and any specific scheduling constraints (needed for second epoch observations), target coordinates and magnitude, the minimal coronagraphic exposition duration, the maximum air mass, the maximum proportion (in time) of the observation that is allowed either before or after the meridian crossing, the minimum exposure time, and the amount of FoV rotation during the observation. It should be noted that the amount of FoV rotation depends on the target 
Table 1. Exoplanets and brown dwarf companions observed during the SHINE campaign.

\begin{tabular}{|c|c|c|c|c|c|c|c|c|c|}
\hline Name & $\mathrm{SpT}_{\text {host }}$ & $\begin{array}{c}M_{\star} \\
\left(M_{\odot}\right) \\
\end{array}$ & $\begin{array}{c}a \\
(\mathrm{au}) \\
\end{array}$ & $\begin{array}{c}\text { Mass } \\
\left(M_{\text {Jup }}\right)\end{array}$ & $\begin{array}{c}\text { Observation } \\
\text { dates }^{(a)}\end{array}$ & $\begin{array}{c}\text { Contrast } \\
(\mathrm{H} 2)\end{array}$ & $\begin{array}{c}\text { Separation } \\
\text { (mas) }\end{array}$ & $\begin{array}{c}\text { PA } \\
(\mathrm{deg})\end{array}$ & Refs. \\
\hline \multicolumn{10}{|c|}{ SHINE discoveries } \\
\hline HIP 64892 B & B9 & 2.09 & $147-171$ & $29-37$ & 2016-04-01 & $7.24 \pm 0.08$ & $1270.5 \pm 2.3$ & $311.68 \pm 0.15$ & (1) \\
\hline HIP 65426 b & $\mathrm{A} 2$ & 1.96 & $80-210$ & $7-9$ & $2016-05-30$ & $10.73 \pm 0.09$ & $830.4 \pm 4.9$ & $150.28 \pm 0.22$ & (2) \\
\hline \multicolumn{10}{|c|}{ Previously known companions } \\
\hline$\eta$ Tel B & A0 & 2.00 & $125-432$ & $20-50$ & $2015-05-05$ & $6.99 \pm 0.22$ & $4214.5 \pm 22$ & $167.25 \pm 0.08$ & $(8)$ \\
\hline CD -352722 B & M1 & 0.56 & $74-216$ & $23-39$ & $2015-29-11$ & $5.89 \pm 0.18$ & $2987.5 \pm 3.51$ & $241.9 \pm 0.06$ & (9) \\
\hline HIP 78530 B & B9 & 1.99 & $\sim 620$ & $19-26$ & $2015-05-04$ & $8.05 \pm X X$ & $4537.5 \pm 3.0$ & $139.98 \pm 0.04$ & $(18)$ \\
\hline$\beta$ Pic b & A3 & 1.61 & $8.5-9.2$ & $9-16$ & $2018-12-15$ & $9.86 \pm 0.1$ & $177.4 \pm 4.51$ & $29.07 \pm 0.6$ & $(3,10)$ \\
\hline HR 8799 b & A5 & 1.42 & $62-72$ & $5.3-6.3$ & 2014-07-13 & $13.19 \pm 0.16$ & $1721.8 \pm 6.0$ & $65.76 \pm 0.19$ & $(4,11)$ \\
\hline HR $8799 \mathrm{c}$ & A5 & 1.42 & $39-45$ & $6.5-7.8$ & $2014-07-13$ & $11.99 \pm 0.16$ & $947.7 \pm 4.0$ & $326.58 \pm 0.24$ & $(4,11)$ \\
\hline HR $8799 \mathrm{~d}$ & A5 & 1.42 & $24-27$ & $6.5-7.8$ & 2014-07-13 & $12.00 \pm 0.17$ & $658.0 \pm 5.0$ & $216.35 \pm 0.52$ & $(4,11)$ \\
\hline HR 8799 e & A5 & 1.42 & $14-17$ & $6.5-7.8$ & 2014-07-13 & $11.93 \pm 0.17$ & $386.1 \pm 9.0$ & $268.81 \pm 1.3$ & $(4,19)$ \\
\hline HD 95086 b & A8 & 1.55 & $28-64$ & 2-9 & 2015-03-02 & $12.37 \pm 0.16$ & $622.0 \pm 4.0$ & $148.8 \pm 0.4$ & $(5,12)$ \\
\hline 51 Eri b & F0 & 1.45 & $10-16$ & $6-14$ & 2016-12-12 & $13.96 \pm 0.51$ & $444.8 \pm 10.6$ & $159.4 \pm 2.6$ & $(6,20,13)$ \\
\hline HIP 107412 B & F5 & 1.32 & $6.2-7.1$ & $15-30$ & $2016-09-16$ & $9.67 \pm 0.09$ & $265.0 \pm 2.0$ & $62.25 \pm 0.11$ & $(22,14)$ \\
\hline PZ Tel B & G9 & 1.07 & $19-30$ & $38-54$ & 2014-07-15 & $5.07 \pm 0.61$ & $478.48 \pm 2.10$ & $59.58 \pm 0.48$ & $(7,15,21)$ \\
\hline $\mathrm{AB} P i c \mathrm{~b}$ & K1 & 0.97 & $\sim 250$ & $13-30$ & 2015-02-06 & $8.04 \pm 0.98$ & $5392.1 \pm 13.1$ & $175.26 \pm 0.15$ & (16) \\
\hline GSC 8047-0232 B & $\mathrm{K} 2$ & 0.89 & $190-880$ & $15-35$ & $2015-09-25$ & $7.67 \pm 0.15$ & $3207.7 \pm 2.7$ & $358.82 \pm 1.03$ & (17) \\
\hline GJ 504 b & G0 & 1.15 & $40-50$ & $4-6$ & 2015-05-06 & $14.56 \pm 0.15$ & $1463.7 \pm 3.8$ & $323.1 \pm 0.1$ & $(23,24)$ \\
\hline
\end{tabular}

Notes. ${ }^{(a)}$ We only list one selected epoch for each target.

References. SHINE papers: (1) Cheetham et al. (2018); (2) Chauvin et al. (2017b); (3) Lagrange et al. (2019); (4) Zurlo et al. (2016); (5) Chauvin et al. (2018); (6) Samland et al. (2017); (7) Maire et al. (2016a); (20) Maire et al. (2019); (24) Bonnefoy et al. (2018), Discovery papers; (8) Lowrance et al. (2000); (9) Wahhaj et al. (2011); (10) Lagrange et al. (2009); (11) Marois et al. (2008); (12) Rameau et al. (2013a); (13) Macintosh et al. (2015); (14) Milli et al. (2017a); (15) Biller et al. (2010); (16) Chauvin et al. (2005b); (17) Chauvin et al. (2005a); (18) Lafrenière et al. (2011); (19) Marois et al. (2010); (21) Mugrauer et al. (2010); (22) Delorme et al. (2017b); (23) Kuzuhara et al. (2013).

coordinates and on the actual time and duration of observations. As it also drives the exposure time, we set a maximum exposition time to avoid overly extending the duration of the exposure.

Classical, additional scheduling constraints are applied on all targets: minimal angular distance to the Moon, avoidance of zenith observations. Poor atmospheric conditions may also be taken into account via pointing restrictions, which usually depend on the wind direction and speed, and via magnitude restrictions in the case of non-photometric conditions. Finally, SPOT also takes into account the various overheads and the need for astrometric and spectro-photometric calibrations. The astrometric calibrations were to be observed as much as possible in the first night of each run and the spectro-photometric on the following night. Following optimisation, SPOT returns a schedule and produces the observing blocks (OBs) that can be automatically transferred to P2 (the ESO observing preparation tool).

In practice, we generally request for the coronagraphic data to be obtained while the target is crossing the meridian at least 15 min before and after the meridian passage, and with at least 30 degrees of FoV rotation, unless more than $7200 \mathrm{~s}$ is required. The minimum exposure time was set to $3600 \mathrm{~s}$. The scheduling of the targets was always optimal; the efficiency of the night could be as high as $100 \%$ when two to three times more targets were available than actually those that were scheduled. However, when not enough targets were available as inputs, short (typically $30 \mathrm{~min}$ ) holes could be present in the schedules during the nights. These holes were used to observe fillers requiring short exposure times (binaries, stars with RV trends, astrometric, and spectrophotometric standards). By making a comparison with other surveys executed in service, such as BEAST (Janson et al., in prep.), we conclude that the use of SPOT has allowed for an increase of more than $30 \%$ in the field rotation angles for identical observing times with respect to the service scheduling routinely applied at ESO.

\subsection{Observing conditions and data quality}

The SPHERE Instruments are fed by an extreme adaptive optics (AO) system called SAXO. It delivers a very high Strehl ratio, which achieves above $90 \%$ in the $H$-band for very good observing conditions by correcting both perturbations induced by the atmospheric turbulence and from the internal aberrations of the instrument itself. A comprehensive description of the SAXO design can be found in Fusco et al. (2016) and Beuzit et al. (2019). Here, we derived the overall statistical AO telemetry data from SAXO and from the ESO MASS-DIMM measurements for the survey observations and relate these parameters to the Strehl ratio, raw contrast, and processed contrast in order to evaluate the performance constraints from these observations. The telemetry data points are spread over 130 different nights and cover more than 200 different observations. The AO telemetry data (available for a large number of our survey observations) includes estimates, from the real-time computer (called SPARTA), several quantities of interest that could be related to the final performances (hereafter SPARTA data) such as: the Strehl ratio and additional atmospheric parameters including the seeing and the 

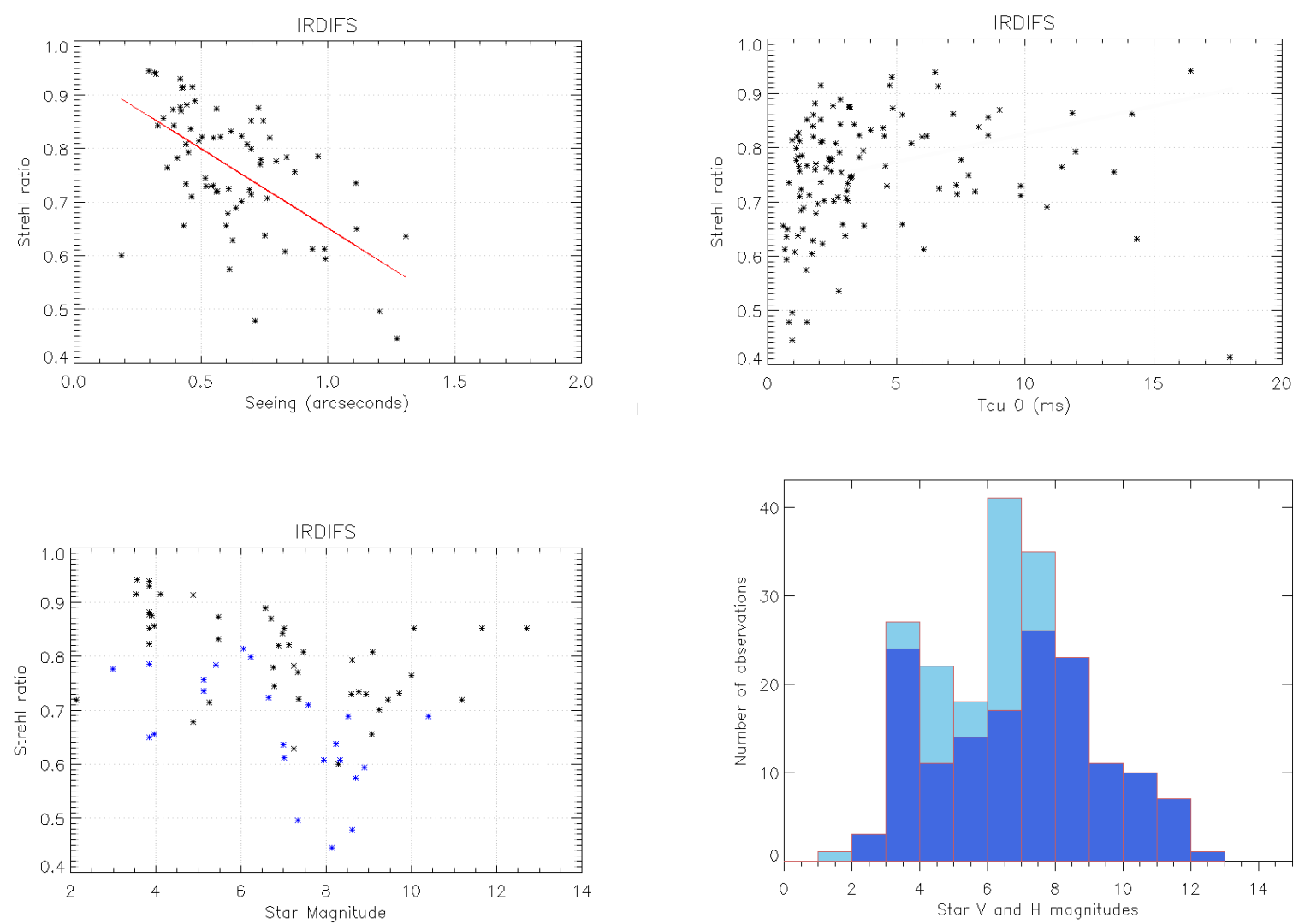

Fig. 1. Observing conditions and AO performances in IRDIFS observing mode: Strehl ratio as a function of the seeing measured by SPARTA (top-left). Strehl ratio as function of the atmospheric coherence time estimated by SPARTA (top-right). A linear fit is overlaid in red in the top figures. Strehl ratio as function of the star $V$ magnitude (bottom-left). The blue data points represent the data taken with coherence time smaller than $3 \mathrm{~ms}$. Histogram illustrating the repartition of the observations (including multiple observations of the same target) as function of the star magnitude in $V$, shown in dark blue, and $H$-bands, shown in light blue (bottom-right). The apparent high number of observations of $R=3.5$ stars is an artificial effect that is due to the high number of observations targeting Beta Pictoris.

coherence time. The Strehl is defined at $1.6 \mu \mathrm{m}$, while the seeing and coherence time are defined at $500 \mathrm{~nm}$.

These quantities are also connected here to the brightness of the target ( $V$ and $H$ magnitudes), retrieved from the Simbad database and used as a proxy in $V$-band for the number of photons received by the wavefront sensor (WFS) ${ }^{1}$. The distribution in magnitude for the sample considered here (for targets with telemetry measurements) is shown in Fig. 1 (bottom-right). It illustrates the fact that our target selection, as described in Desidera et al. (2021), was based on setting a magnitude limit $(V<12.5)$ in order to guarantee good AO performances. Our sample selection criteria also favors red targets, leading to the faintest $H$-band observations at below a magnitude of eight.

The overall good AO performance for the selected range of target brightness is confirmed by the Strehl measurements shown in Fig. 1 (bottom-left). This figure shows the distribution of the Strehl ratio as a function of the star magnitude in $V$-band where the median Strehl is greater than $70 \%$, averaging around $80 \%$ in moderate seeing conditions. This figure also shows a decrease in the Strehl beyond a magnitude of seven. The blue data points,

1 For stars with a magnitude $R<10$ (value read from the SPHERE acquisition template), the WFS arm uses a red filter called $L P 780$ blocking wavelengths below $780 \mathrm{~nm}$. As a result the central wavelength of the WFS arm is shifted to redder wavelengths, approximately $850 \mathrm{~nm}$, and the nearest broadband filter is the $I$ filter $\left(\lambda_{\mathrm{c}}=806 \mathrm{~nm}\right.$, $\Delta \lambda=149 \mathrm{~nm}$ ). However, not all stars have a measurement of their I magnitude in Simbad, we therefore used the $V$ mag which is most commonly available. representing a coherence time that is smaller than $3 \mathrm{msec}$, highlight the impact of short coherence times on the performance with a Strehl ratio below $80 \%$. Milli et al. (2017b) suggest that the Strehl or its estimation could be affected by the small number of photons reaching the WFS (for 7.5-9 magnitude guide stars), while the AO loop is operating at full speed, which could explain the Strehl decrease around a magnitude of eight. For fainter stars, a slower AO loop frequency of $600 \mathrm{~Hz}$ is used in order to still collect a sufficient number of photons per $\mathrm{AO}$ cycle for high-order wavefront determination and correction.

This hypothesis is supported further by the greater decrease in Strehl for the low-coherence time data points, which explain most of the performance inflection around $V=8$ magnitude. The large scatter in the Strehl distributions indicates that the seeing is also, as expected, a parameter influencing the Strehl among others such as $\tau_{0}$ and the star magnitude. Despite this large scatter, the (top-left) plot from the same figure shows a linear decrease trend of the Strehl with the seeing. This decrease is on average $1.8 \%$ Strehl for an increase of $0.1^{\prime \prime}$ in seeing, which is similar to the Strehl behavior versus seeing obtained by Milli et al. (2017b) when the correction is applied according to the SPARTA versus DIMM measurements dependency. While the SPARTA seeing provides an estimate that is closer to the image quality in the science frames, it has been shown that the SPARTA seeing estimations are smaller than the ESO DIMM measurements, a fact that may be accounted for the turbulence outer scale. The overall impact of the performance limitation from the seeing on our survey is clearly important, as illustrated by the large number 
of observations occurring for seeing greater than $0.8^{\prime \prime}$ (Fig. 1, top-left).

The top-right part of Fig. 1 shows the distribution of the Strehl as a function of the coherence time for our full sample. There is a steep rise in Strehl ratio with the coherence time (for $\tau_{0}<3 \mathrm{~ms}$ ) followed by plateau for larger coherence times (for $\tau_{0}<5 \mathrm{~ms}$ ). This shows that for low coherence times, the AO performances are limited by the temporal bandwidth error as mentioned in Cantalloube et al. (2020), who also derive a $3 \mathrm{msec}$ threshold from the point of view of the system. This is in agreement with the laboratory and first on-sky measurements described in detail in Petit et al. (2012). The overall impact of this performance limitation on our survey is likely to prove important, as illustrated by the large number of observations occurring when $\tau_{0}<4 \mathrm{~ms}$ (i.e., $70 \%$ of the observations). A further analysis of the impact of this effect should include the seeing contribution in the Strehl error budget in order to disentangle the seeing and coherence time correlations.

In summary, we show here that the AO performances are clearly related to the observing conditions for our sample. We further describe the impact of these observational parameters on the high-contrast performance in Sect. 4.

\section{Data reduction and analysis}

Large surveys such as SHINE (especially given the large IRDIS FoV) unveiled a high number of point sources (mainly background sources) from which true sub-stellar mass companions need to be distinguished. For this reason, both the astrometry and photometry of the detected point sources need to be precisely and homogeneously calibrated and extracted to test their companionship and charactezerize their nature. We detail, in the following subsections, the SHINE strategy: (i) to calibrate both IRDIS and IFS on various important aspects (distorsion, plate scale, true north, parallactic angle determination, central start position) in Sect. 3.1; (i) to pre-process the scientific observations; (ii) apply basic reduction steps (cosmetics, flat, frame registration and recentering, cross-talk and wavelength calibration) as described in Sect. 3.2; (iii) to apply advanced ADI/ASDI algorithms summarized in Sect. 3.3; and (iv) ultimately extract the companion relative astrometry and photometry as detailed in Appendix A, which includes the final error budget for both the astrometry and photometry.

\subsection{SPHERE calibration}

\subsubsection{Astrometry}

In this section, we present the astrometric calibration of the SHINE survey which depends on several factors described in detail in the following paragraphs: (i) platescale, true north, and distorsion correction; (ii) angular offset (between field and pupiltracking and parallactic angle correction); (iii) star-centering; this section also presents, as a conclusion, the (iv) sanity check of the calibration startegy based on Gaia-DR2 results.

Platescale, true north, and distortion. An extensive description of astrometry with SPHERE can be found in Maire et al. (2016b) and Beuzit et al. (2019). In what concerns the SHINE survey in particular, the astrometric calibration consists of the correction for the instrument anamorphism $(0.60 \pm 0.02 \%$ between the horizontal and vertical directions of the detector, i.e., 6 mas at 1 arcsec), correction for constant offset angles (between the IFS and IRDIS fields of view, between pupiltracking mode and field-tracking mode), and a determination of the values for the pixel scale, as well as the correction to true north. We find small but non-negligible variations of the last quantities with time, requiring dedicated calibration for each observing run. Appropriate values were estimated for each run using several reference fields of view in clusters with a high number of stars and having accurate (sub-mas) astrometry from Hubble Space Telescope (HST) or ground-based diffraction limited observations: 47 Tuc (Bellini et al. 2014, 2017; Soto et al. 2017); NGC 6380 (Bellini et al. 2011; Soto et al. 2017) and Noyola (priv. comm.); NGC 3603 (Harayama et al. 2008; Khorrami et al. 2016; Rochau et al. 2010); and Trapezium B1-B4 (Close et al. 2012, 2013) (see Fig. 2).

IRDIS was used for all astrometric calibrations because its field of view allows for the observation of a large number of stars, in most cases, between 50 and 100. Only seven stars were available for the Trapezium B1-B4 field, leading to a less accurate calibration of the field orientation (by 1\%) and on the plate scale (by $0.1 \%$ ). It was used only when the other calibration fields were not accessible from February 2015 to March 2016. Due to the reduced accuracy of the catalog positions for NGC 6380 and the smaller number of calibrating objects, we used this field only twice between May-June 2015 and once in June 2017. The use of several calibrators enabled us to perform calibrations throughout the year and to do cross-calibration. Because the coronagraph has a small effect on the pixel scale, the astrometric fields were observed with a coronagraphic plate, including an offset for the Trapezium B1-B4 field observation to shift the B1 star out of the coronagraphic mask.

The pixel scale is slightly different for the $H 2, H 3, K 1$, and $K 2$ filters, with mean values of $12.255,12.250,12.267$, and 12.263 mas pixel $^{-1}$, respectively. For IFS, we used a constant value of the pixel scale of $7.46 \pm 0.02$ mas pixel $^{-1}$. Variations in this case have less impact on the results due to the small field of view. The typical measurement accuracy of the pixel scale is \pm 0.012 mas pixel $^{-1}$ using our two best fields 47 Tuc and NGC 3603, while the true north correction (weighted value 1.77 degree) typically has an error of \pm 0.07 degree. For IFS, an additional offset of 100.48 degree in the clockwise direction is applied to account for the orientation of the instrument FOV. This leads to uncertainties in the position of 3-4 mas at the edge of the IRDIS field of view and less than 1 mas for IFS. For comparison, typical accuracy of GPI using their best calibrations are \pm 0.021 mas pixel $^{-1}$ in the pixel scale and \pm 0.12 degree in the true north correction (De Rosa et al. 2020). The improved calibration accuracy obtained for SPHERE is due to the wider field of view of IRDIS that allows for the use of stellar cluster fields as calibrators with more accurate catalog positions.

Field and pupil tracking and parallactic angle calibration. While the SHINE science observations are taken in pupiltracking mode for ADI purposes, the astrometric fields were observed in field-tracking mode. The offset between the pupiltracking and field-tracking modes was measured at the beginning of the survey to be equal to $-135.99 \pm 0.11$ degree. The parallactic angle is computed from the data FITS header: timestamp, RA/Dec of the derotator (INS4.DROT2.RA/Dec), which are more accurate than the "RA" and "Dec" keywords (J2000 coordinates of the target). When these parameters are used, it is possible to correct for the precession of coordinates between 2000 and the date of observation. In order to derive the precise parallactic angle of each DIT from the timestamp we also included a parametric model of the overheads. We observe a systematic error in the parallactic angle estimation due to backlash in the derotator mechanism of $\sim 0.05^{\circ}$, as demonstrated in 

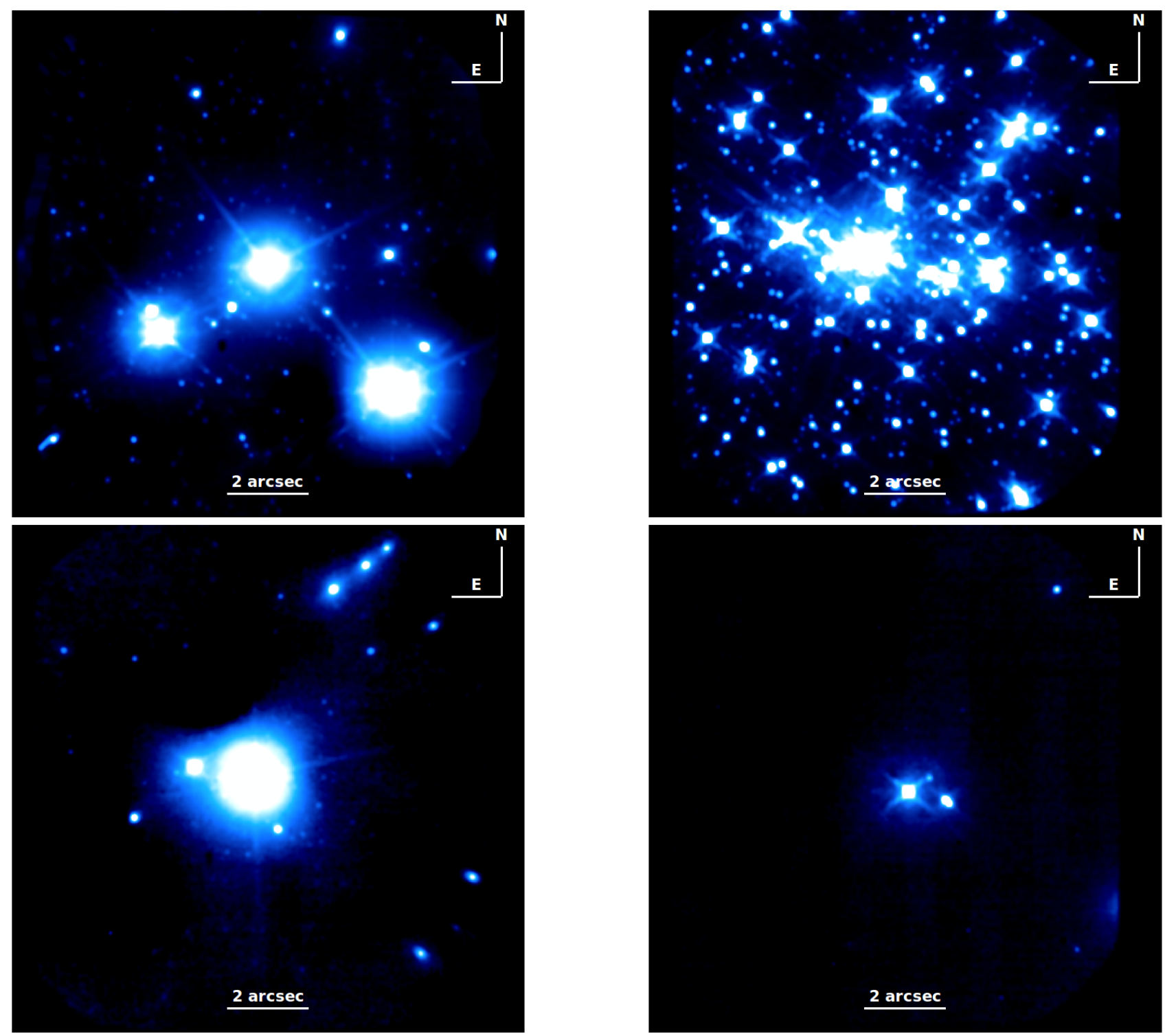

Fig. 2. IRDIS H2 images of the fields used for the astrometric calibration. Upper left panel: 47 Tuc. Upper right panel: NGC 3603. Lower left panel: NGC 6380. Lower right panel: Orion B1B4. A logarithmic intensity scale is used to show also faint stars.

Beuzit et al. (2019). In pupil-stabilized mode, this leads to a $\sim 0.4$ pixel difference in the position of an object located at the edge of the IRDIS FoV on either side of the meridian.

Star centering. The astrometry we consider in this paper is relative to the star. Since the star point spread function (PSF) peak is hidden by the coronagraphic mask and to avoid concerns related to the non-uniform intensity distribution of the coronagraphic leakage, its position was determined using a special calibration (STAR-CENTER), where four faint replicas of the star image are created by giving a bi-dimensional sinusoidal profile to the deformable mirror (see Beuzit et al. 2019; Makidon et al. 2005). The STAR-CENTER calibration was repeated before and after each science observation and the center estimations of the results were averaged. While this calibration greatly reduces uncertainties with regard to the exact position of the star and the center of the field rotation, experience shows that small drifts of a few mas of the star center during long sequences of $\sim 1-2 \mathrm{~h}$ can occur. For this reason, in order to increase the star centering accuracy, we generally used, for the second epochs when carrying out (candidate) companion follow-ups, the STAR-CENTER setup solely for the science exposure.

We performed specific measurements to estimate the accuracy of the central star position when the STAR-CENTER setup is not used for the science exposure (i.e., most of the cases presented in this paper). To do so, we measured the position of the central diffraction peak on the IFS datacubes collapsed in wavelengths and making the mean over all detector integration time (DIT); as mentioned above, errors are likely independent of the errors in the STAR-CENTER procedure. We found that the mean position of the center is offset with respect to the nominal position along the left-right direction in the pupil reference frame by a small, but significant amount $0.52 \pm 0.10$ mas for Y-H observations, while there is no offset for the $Y-J$ mode $(0.03 \pm 0.05$ mas $)$ or along the top-bottom direction in the pupil reference frame in both modes. The root mean square (rms) scatter of the residuals after a $3 \sigma$ clipping are 1.35 mas (1.23 mas) in RA and 1.24 mas (1.43 mas) in declination, for the Y-H $(Y-J)$ mode. Most outliers are found in data sets that were not validated and a few of them are binaries. There are $5 \%$ of the validated observations that have 
much larger dispersion of the position of the peak than usual; these anomalous cases make $\sim 10 \%$ of the observations acquired before February 2016, while the fraction is reduced to $\sim 2 \%$ after that epoch. This is likely due to improvements in the AO calibration that resulted in less distorted diffraction peaks. A few of these residual cases may be unresolved binaries. We conclude that a reasonable estimate for the accuracy of the absolute central star position is \pm 1.5 mas for both IFS and IRDIS.

Cross-check with Gaia DR2. An external check of the accuracy of our astrometry is provided by wide companions in the Gaia Data Release 2 catalog (Gaia Collaboration 2018). The number of sources in the IRDIS field of view with a contrast adequate to be detected with Gaia is limited because stars with known bright companions were not included in our sample with the aim of avoiding problems for the $\mathrm{AO}$ and heavy saturation of the detector. By comparison with the SPHERE results, we find that Gaia DR2 limiting contrast (in the visual $G$-band) corresponds to contrasts in the near-infrared of $\Delta H=2.5$ mag for separation sep $<2.5 \operatorname{arcsec}$ and 6 mag for sep $>4 \operatorname{arcsec}^{2}$. For this comparison, we considered 34 IRDIS close-companion candidates (some of them are not included in the sample described in this paper), with $\Delta H 2<6 \mathrm{mag}$; out of which twelve are in Gaia DR2. We did not considered one of the Gaia DR2 data because it has very large error bars. In addition we removed some SHINE objects because they are saturated or located at the edge of the IRDIS FOV ( $\operatorname{sep}>6.5$ arcsec), both leading to inaccurate astrometry. Gaia measurements are most likely unreliable for very high contrasts. This is the case of HD $1160 \mathrm{C}$, for which the Gaia contrast is $8.24 \mathrm{mag}$. Binary periods for these stars are so long that they should not affect the result. However, a couple of the objects are not binaries but rather background stars (HIP 82430 and PDS 70). We took into account the relative proper motion between the SHINE observation and the Gaia epoch (2015.5). At the end we have seven good comparative measurements. The separation measured by SHINE is slightly larger than that measured by Gaia DR2. The mean offset is $-2.8 \pm 1.5$ mas, with a root mean square (rms) of 3.9 mas. The position angle measured by SHINE is similar to that measured by Gaia DR2: the mean offset is $0.06 \pm 0.04$ degree, with an $\mathrm{rms}=0.11$ degree. The rms agrees well with the expected uncertainties in these quantities in the SPHERE data.

We conclude that the minimum accuracy of the astrometric calibration of SHINE data is \pm 2 mas at separations $<1$ arcsec, and \pm 3 mas at larger separations. These values are similar to the scatter typically observed for GPI astrometric calibrators (De Rosa et al. 2020). In practice, the astrometric accuracy on the position of faint substellar companions detected in the SHINE data is limited by the measurement uncertainties from the image post-processing (Sect. 3.3) and by the companion magnitude.

\subsubsection{Photometry}

Below, we describe the strategy for deriving the relative photometry of the SHINE candidates considering the unsaturated and coronographic observations of a scientific target, along with two tests done by using IFS to validate our strategy for both IRDIS and IFS, which have very similar photometric biases.

Strategy. In the SHINE survey, photometry of candidate companions and limiting magnitudes for non-detections are relative in contrast to the central star. Since the star is behind

\footnotetext{
2 Since the companions are typically much redder than the central star, the contrast in the visual band is a few magnitudes deeper.
}

the coronagraphic mask, simultaneous photometry is not possible. We thus include a flux calibration (STAR-FLUX) for both IRDIS and IFS that is acquired just before and after the science exposure by offsetting the differential tip-tilt stage (DTTS) by about 0.5 arcsec with respect the coronagraphic mask using the SPHERE tip/tilt mirror (Beuzit et al. 2019). When performing this calibration, suitable neutral density filters are inserted to avoid detector saturation. The transmissions of these neutral density filters were carefully calibrated from 0.9 to 2.3 microns (see Beuzit et al. 2019 and the ESO website ${ }^{3}$ ) and are taken into account for the contrast estimation. This procedure works very well in stable conditions, but it may be affected by variations of the Strehl ratio from evolving observing conditions. Higher photometric accuracy can be achieved by using the waffle pattern continuously (for companion candidates follow-up) during the observing sequence to monitor the Strehl variations.

Cross-check with catalogues and binary companions. A first test of the photometric calibration accuracy is provided by comparing the peak counts of the diffraction image of the flux calibration (corrected for the integration time and for the neutral density filter transmission) to the apparent magnitude of the stars from the 2MASS catalogue (Skrutskie et al. 2006), corrected for atmospheric extinction. This procedure neglects stellar variability and the impact of variability on the Strehl ratio. Using $J$-band IFS data, we obtain an rms of residuals of $0.15 \mathrm{mag}$ for observation in the $Y-H$ mode, and 0.19 mag for those in the $Y$-J mode. This has been computed after eliminating the expected variations due the Strehl ratio and after clipping outliers deviating greater than 2.5 standard deviation from the mean with an iterative procedure (about $12 \%$ of the data). A similar value is obtained by considering the rms scatter of the estimate of the coronagraph central transmission, measured as the ratio between the maximum counts of the diffraction peak in the coronagraphic image and in the flux calibration (corrected for the length of the integration and neutral density filter transmission). The average coronagraphic transmission measured in this way are $0.00168 \pm 0.00005$ for the $Y-H$ mode, and $0.00208 \pm 0.00001$ for the $Y-J$ mode, respectively, for a median SHINE observation.

A second test of the photometric calibration accuracy is provided by the photometry of three standard systems (HD 2133, HD 114174, and REJ1925-563), composed of a main sequence star and a white dwarf with separations in the range of $0.2-$ 0.7 arcsec that we observed during the survey. These systems were observed several times, with typically at least one observation per run. For schedule optimization, these observations were acquired in ADI with the star quite far from meridian and in poorer observing conditions than typical survey targets: here, we consider only those observations that were obtained in fair to good atmospheric conditions. The photometry of the faint companions was obtained by inserting a negative scaled PSF at the position of the companion in the raw datacube, that is, before combining the various DITs. We then measured the rms residuals of the differential image obtained through monochromatic principal component analysis (PCA: Soummer et al. 2012) around the companion. The procedure then minimizes these residuals by simultaneously adjusting the contrast and the position. The values we consider are the mean of the results obtained using 2-6 PCA modes. The average contrasts in the $J$-band obtained with this procedure using the main sequence star and the white dwarfs are 7.68, 10.04, and $6.10 \mathrm{mag}$ for HD 2133, HD 114174, and REJ1925-563, respectively. The rms value for the contrasts

\footnotetext{
3 https://www.eso.org/sci/facilities/paranal/

instruments/sphere/inst/filters.html
} 

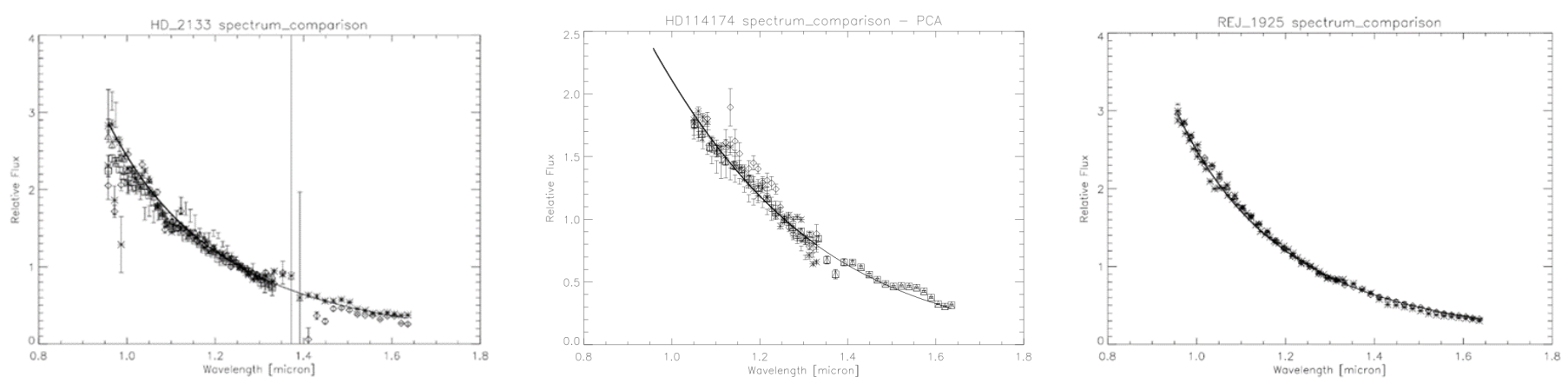

Fig. 3. Spectra of the white dwarfs used as spectrophotometric standards compared with predictions from model atmospheres (solid lines). Different symbols are results obtained from different epochs. We normalized the observed spectra at the median flux in a narrow range around 1.25 micron.

in the $J$-band $/ H$-band are $0.11 / 0.05,0.18 / 0.08$, and $0.05 / 0.07 \mathrm{mag}$ for HD2133 (8 observations), HD 114174 (14 observations), and REJ1925-563 (6 observations). The photometric errors increase, as expected, with magnitude; as a consequence these errors are smaller in $H$-band than in $J$-band. This may be also attributed to a higher impact of the variation of the SR and of the speckle noise for fainter targets and shorter wavelengths. A comparison of the spectra determined by our method with atmospheric models is given in Fig. 3.

We conclude from these measurements that a reasonable photometric errors estimate for both the limiting contrast and for companions characterisation (not including the ADI postprocessing error contribution) is around $\pm 0.2 \mathrm{mag}$. It is dominated by the STAR-FLUX variation, which is also the case for the survey data photometric error that is, on average, equal to $\pm 0.25 \mathrm{mag}$.

\subsection{SPHERE data center preprocessing}

\subsubsection{IRDIS-only steps}

The SHINE survey was reduced by the SPHERE Data Centre (hereafter, SPHERE-DC) ${ }^{4}$. For the IRDIS data, the first reductions steps (dark/background, flat, and bad pixel correction) rely on the SPHERE data reduction and handling (DRH) pipeline (Pavlov et al. 2008) provided by ESO. We used the on-sky background because there is a significant difference between day-time background calibrations and the on-sky background recorded in the science frames. This is the case mostly in the $K$-band with a systematic, spatially variable offset (typically of the order of $100 \mathrm{ADU}$ for a $64 \mathrm{~s}$ exposure time), which is due to the sky background contribution itself. A similar effect is also visible in $H$-band, on a smaller scale that is typically only a few ADU.

Since most of the SHINE observations use the pupil-tracking observing mode, a very accurate determination of the star center is needed in order to successfully use both the angular differential imaging and spectral differential imaging methods. Also, since IRDIS is used in dual-band imaging, the star center in both IRDIS channels is used to combine them. By default, we use the DRH sph-ird-star-center routine to determine the star center position, using the waffle images acquired for this purpose just before and after the science observations. This very fast routine provides an accurate centering in many cases but it could fail for weak waffle spots, especially in the $K$-band, where these spots can be hidden beneath a much stronger thermal background noise; this can also be the case when the deformable

\footnotetext{
4 http://sphere.osug.fr/spip.php?rubrique16\&lang=en
}

mirror offsets to create this pattern are set too low. We therefore designed an automated way to check the quality of the DRH centering by comparing the two center positions derived from the four waffles. When the distance between these two possible center positions was found to be greater than a 0.9 pixel, we used a SPHERE-DC-made IDL routine that is more robust to identify weak waffle spots. This routine is able to detect weaker waffles by locating them within small circular apertures located at the expected (wavelength-dependent) position and by using a combination of a high-pass spatial filtering, sky-background subtraction, and median stacks of all waffle images available in order to increase their signal-to-noise ratios (S/Ns).

A small fraction of the SHINE datasets use continuous waffle mode observations, meaning that the waffle spots are activated during the entire observation for better astrometric monitoring (with some localized loss in the limiting contrast because of the secondary spots). In these specific cases, we performed an individual re-centering of each frame in the sequence using the SPHERE-DC dedicated star-centering routine described above. This improves the quality by correcting any drift or jitter of the targeted star behind the coronagraph and, as a result, this improves the quality of the astrometric measurements by removing these sources of error.

\subsubsection{IFS-steps}

For the IFS data, we used the data reduction and handling (DRH Pavlov et al. 2008) pipeline, however, we complemented it with additional steps implemented at the SPHERE Data Center (Mesa et al. 2015; Delorme et al. 2017a) to improve the wavelength calibration, to apply a correction for cross-talk, and to improve the handling of bad pixels. The improvements of the wavelength calibration are obtained by using a cubic fit whose coefficients are estimated from the wavelength shifts of the spots generated by the STAR-CENTER calibration, which are known to scale linearly with wavelength. The cross-talk correction is performed using an iterative procedure that corrects for the spectrograph PSF, using coefficients derived using appropriate tests performed in the laboratory during the instrument assembly. Bad pixels are corrected using a dedicated sky observation acquired at the end of each science exposure. This procedure yields more accurate results than the one based on the flat field calibration within the DRH.

\subsubsection{Final steps common to IRDIS and IFS}

After these pre-reduction steps, both IRDIS and IFS datasets are corrected for the instrument anamorphism and the astrometric solution (pixel scale and true north) estimated from the 
calibration, described in Sect. 3.1.1, is applied to the dataset. The output data is composed of a pre-reduced master cube combining all frames obtained during a given observation sequence, that is used as input for all angular differential imaging (ADI Marois et al. 2006) algorithms. We also associate to this master cube a vector of de-rotation angles for each frames, using the accurate timing and overheads for each frame, to produce a frame-to-frame determination of the parallactic angle.

\section{3. $A D I$ and $A S D I$ postprocessing}

For both IRDIS and IFS, we obtained good-quality recentered images gathered into a single master cube associated with their parallactic angle values. Subsequent steps follow to estimate and subtract the stellar halo from each images, followed by derotation and stacking of the residuals. The most critical step is the estimation of the stellar halo that drives the level of the noise residuals. We applied different angular differential imaging (ADI) algorithms to optimize the detection performances and to identify associated biases. We rely mainly on the SpeCal (Galicher et al. (2018) software which offers various ADI options. For the homogeneous reduction, we selected TLOCI (Marois et al. 2014; Galicher et al. 2018) and then the PCA (Soummer et al. 2012) for IRDIS and PCA angular and spectral differential imaging (ASDI: Mesa et al. 2015) for IFS.

In the TLOCI approach (Lafrenière et al. 2007), the PSFreference is estimated for each frame and each location. Linear combinations of all data are computed to minimize the residuals into an optimization zone, which is much bigger than the subtraction zone, to avoid the self-removal of point-like sources. The SpeCal version of the TLOCI algorithm is derived from the one described in Galicher et al. (2018), assuming a flat planet spectrum in contrast. Adjustable parameters are used to select the frames and to describe the regions of interest. In SpeCal, the gap between this region and the region of interest is set to 0.5 full width half-maximum (FWHM). Hence, the optimizing region is far enough from the region of interest so that the flux of a source in the latter does not significantly bias the linear combination. Finally, an additional parameter sets the radial width of the optimizing region. Here, we considere a radial width of the subtraction zone of 1 FWHM in radius; a radial-to-azimuthal width ratio of 1.5; a standard surface of the optimization zone was $N=$ 20 PSF FWHM and $10 \%$ is used for the minimum residual flux ratio due to self subtraction compared to the flux of a putative candidate.

For historical reasons, two PCA algorithms are implemented in SpeCal. The first version can be applied on IRDIS or IFS data using ADI or ASDI. This algorithm follows the equation of Soummer et al. (2012). In the ADI case, which is the option selected to reduce IRDIS data in this paper, the principal components are calculated for each spectral channel independently. Each frame is then projected onto a limited number of modes. In the ASDI case, the algorithm is the same but it works simultaneously on the spatial and spectral frames. The second version of PCA, used to reduce IFS data in this paper, is very similar to the first version we describe earlier in this paper but it was applied only on IFS data using the ASDI option (Mesa et al. 2015). In addition, it is worth mentioning that both PCA algorithms we use have no frame selection for minimizing the self-subtraction of point-like sources when deriving the principal components.

For PCA and TLOCI algorithms that bias the photometry of off -axis point sources, SpeCal estimates the throughput at each position in the field by generating a datacube of fake planets for which the ratio of the flux in the resulting image to the flux of the fake planet is calculated to obtain the centro-symmetrical 1D-throughput as a function of the angular separation which is then applied to the images. For PCA, Specal estimates the throughput by inserting fake planets injections which are only ten times brighter than the local stellar residuals level after PCA. This makes it possible to be close to the level of interest while being sufficiently above the residual to minimize the bias in the throughput estimation. For TLOCI, the throughput is calculated from an analytical formula (see Sect. 2.10.2 of Galicher et al. 2018). The contrast curves, which we derived as described as follows, for each spectral channel are based on the azimuthal standard deviation calculated in annuli of 0.5 FWHM width. Finally, the $5 \sigma$ detection limits are derived by taking into account the flux loss from ADI self-subtractions, the transmission of the coronagraph at short separations (close to the inner working angle (IWA: 0.1") and the transmission of the neutral-density filter if used when registering the PSF. These detection limits are thus normalized by the unsaturated PSF flux. Both 1D and 2D contrast maps were estimated following these steps for each star for each reduction technique.

All target stars were processed for each instrument in a homogeneous way, using at least TLOCI or PCA with similar sets of parameters. We considered 50, 100, and 150 PCA modes for IFS and 5 for IRDIS. We inspected by eye at least three residual maps for each star and for both IFS and IRDIS to look for candidate companions (CC). We also included small number statistic corrections. In addition to the standard SpeCal reductions, we also used, in several cases, ANDROMEDA (Cantalloube et al. 2015) and PACO (Flasseur et al. 2018, 2020b) algorithms to search for points sources. Given their statistical robustness to derive detection limits and to better identify false detections these algorithms go on to become the main algorithms for the final analysis of the SHINE survey.

\subsection{Astrometric and photometric extraction for point sources}

The PCA and TLOCI algorithms are known to distort the images of any off-axis point-like source. To accurately retrieve the relative photometry and astrometry of the detected candidate companions (CC) with their uncertainties, SpeCal fits a model of an off-axis point source image to the detected point source and adjusts its position and flux to locally minimize the flux. After building a model of the point source using the technique described in Galicher \& Marois (2011), the flux and the position of this synthetic image are adjusted within a disk of diameter of a FWHM of three so that it includes the positive and the negative parts of the point source image. To optimize the computation time, instead of calculating the synthetic image each time we test a new planet position, we shift the synthetic planet image to its rough position. Once the optimization is completed, we measure the excursion of each parameters that increases the minimum residual level by a factor of 1.15 . These excursions correspond to the $1 \sigma$ accuracy due to the fitting errors in the SpeCal outputs. The spectrum extraction from IFS data is performed similarly by processing the wavelength channels separately. For the channels with no detection above $1 \sigma$ we provide an estimation of the upper limit only. In conclusion, we summarize the astrometric and photometric error budget given by Specal considering both calibrations and ADI/ASDI extraction errors. For astometry, this budget includes: (1) calibration with uncertainties of the detector distorsion, plate scale, and true north; (2) determination of the correction for constant offset angles (between the IFS and IRDIS fields of view, between pupil-tracking and 

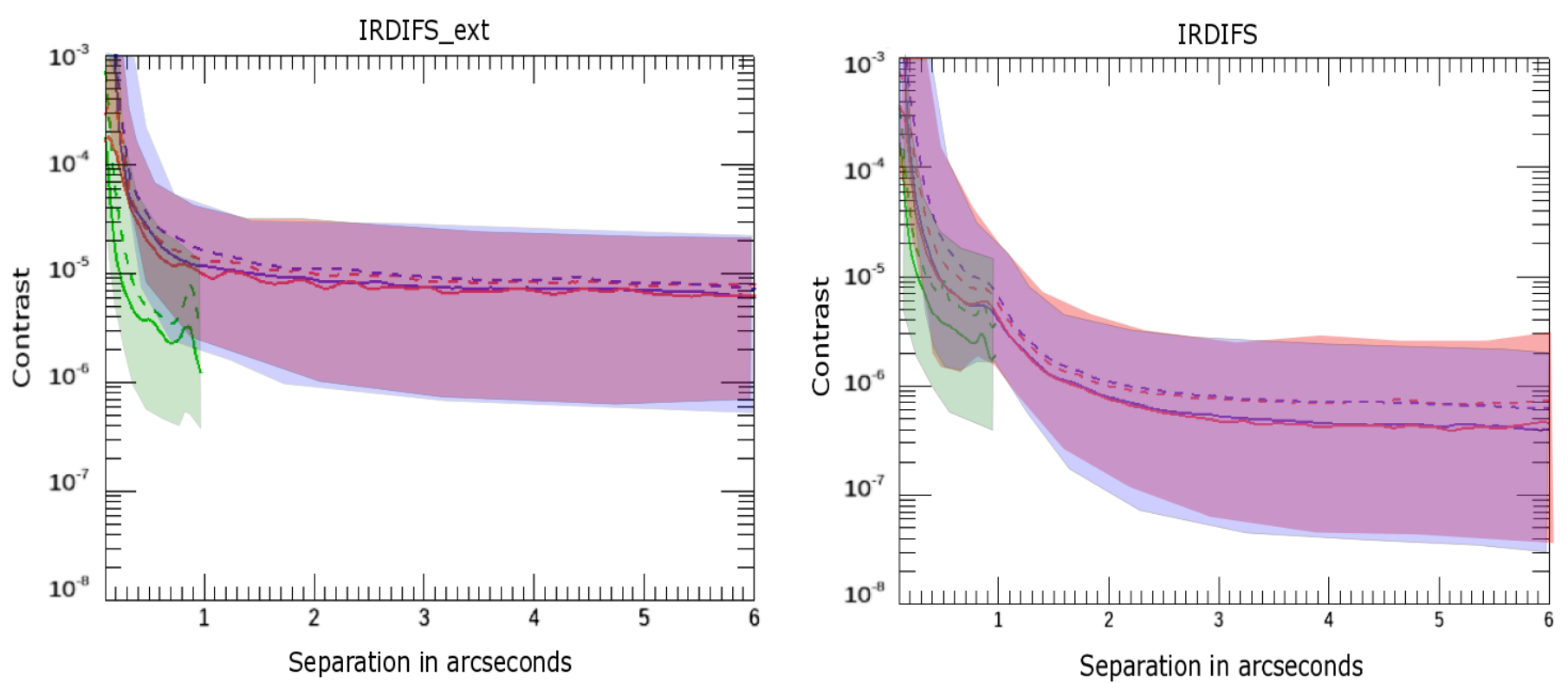

Fig. 4. Contrast curves at $5 \sigma$ obtained for the full sample for irdifs-ext (left) and irdifs (right) modes observations. The solid line gives the median value of the contrast. The dashed line gives the mean value of the contrast. Red indicates IRDIS data reduced in PCA ADI; blue indicates IRDIS data reduced in TLOCI ADI; and green indicates IFS with a PCA ASDI reduction.

field-tracking modes); (3) calculation of the parallactic angle variation (correction of precession and timestamp); (4) central star position; (5) determination of the companion and candidate relative positions with Specal (Galicher et al. 2018).

For photometry, it includes: (1) Contrast estimation with proper calibration of neutral density, exposure time, and associated error related to the variation between Start/End STARFLUX calibrations but not including the strehl variation during the observing sequence (psf); (2) Temporal variations of the stellar coronographic flux between 30-50 pixels during the observing sequence (Seq); (3) Companion flux determination considering ASDI signature, taking into account flux cancellation from the algorithm used, and coronograph attenuation correction as described in Galicher et al. (2018).

\section{High contrast performance}

\subsection{Contrast curves}

We derived the $5 \sigma$ IRDIS and IFS contrast curves of each observation for all the targets in the sample as presented in Figs. 4 and 5. These detection limits are derived based on the noise in the speckle-subtracted image, compensated for the throughput of the algorithm (calibrated with fake planet injections), the transmission of the coronagraph (calibrated from measurements in SPHERE), and the small sample statistics (Mawet et al. 2014). More details are provided in Galicher et al. (2018). Two types of contrast are discussed in detail in this section: the raw contrast, computed on the median coronagraphic image of each observation, and the contrast after post-processing described above. As illustrated in Figs. B.1 and B.4, the raw coronagraphic contrast at various separations shows a strong dependency on the Strehl and seeing, both estimated by the average of the SPARTA values during the coronagraphic sequence. The two smaller separations (from 100 to 700 mas) are within the $\mathrm{AO}$ control radius located at 840 mas separation radius in the $H$-band.

This dependency remains visible in the post-processed data, especially at 500 mas, despite other factors also coming into play, such as the field rotation and the stability of the relative conditions (see Fig. B.2). At small separations, these scatter plots shows that it is possible to easily gain one magnitude in raw contrast by increasing the Strehl by $10 \%$ or the decreasing the seeing by $30 \%$. As a consequence, it is clear that conducting the survey in visitor mode, which does not offer the best seeing conditions, had some impact on the performance of the survey ultimate contrast. Outside the $\mathrm{AO}$ correction radius, at separations greater than 900 mas, there is still a smaller dependency of the contrast on the seeing due to the residual light scattering outside the AO control radius and the lower height of the diffraction peak. As part of this study, the dependency of the post-ADI contrast on parameters that trace the quality and stability of the conditions was also investigated, as illustrated in Figs. B.1, B.4, and B.2. We considered the dispersion in the seeing, coherence time, and Strehl over the duration of the pupil-stabilised sequence. No significant correlation could be drawn from this sample. It is worth noticing that at small separation (100 mas) the processed contrast is lower than the raw contrast because of the very small angular rotation, the small throughput of the algorithm (from self subtraction), and the stronger coronagraphic residuals.

The gain from raw contrast to post-processed contrast is clearly visible in Fig. 6, which shows a typical improvement greater than a magnitude of five at short separations for both IRDIS and IFS. It is worth noticing that the contrast gain in the IFS field of view is consistent for all separations and reaches at least a magnitude of seven. For the shorter separation, located at the edge of the coronagraph (100 mas), there is marginal improvement for IFS from ASDI and no improvement for IRDIS in contrast due to the very small angular rotation, the small throughput of the algorithm and the stronger coronagraphic residuals for both instruments. At larger separations than the AO cutoff, the improvement from the post-processing ranges between magnitudes of two to four.

We also plot in Fig. B.3 the processed contrast as function of the star magnitude for both IFS and IRDIS at various separations. From these figures, it is clear that there seems to be a small correlation between these parameters when the post-processing 

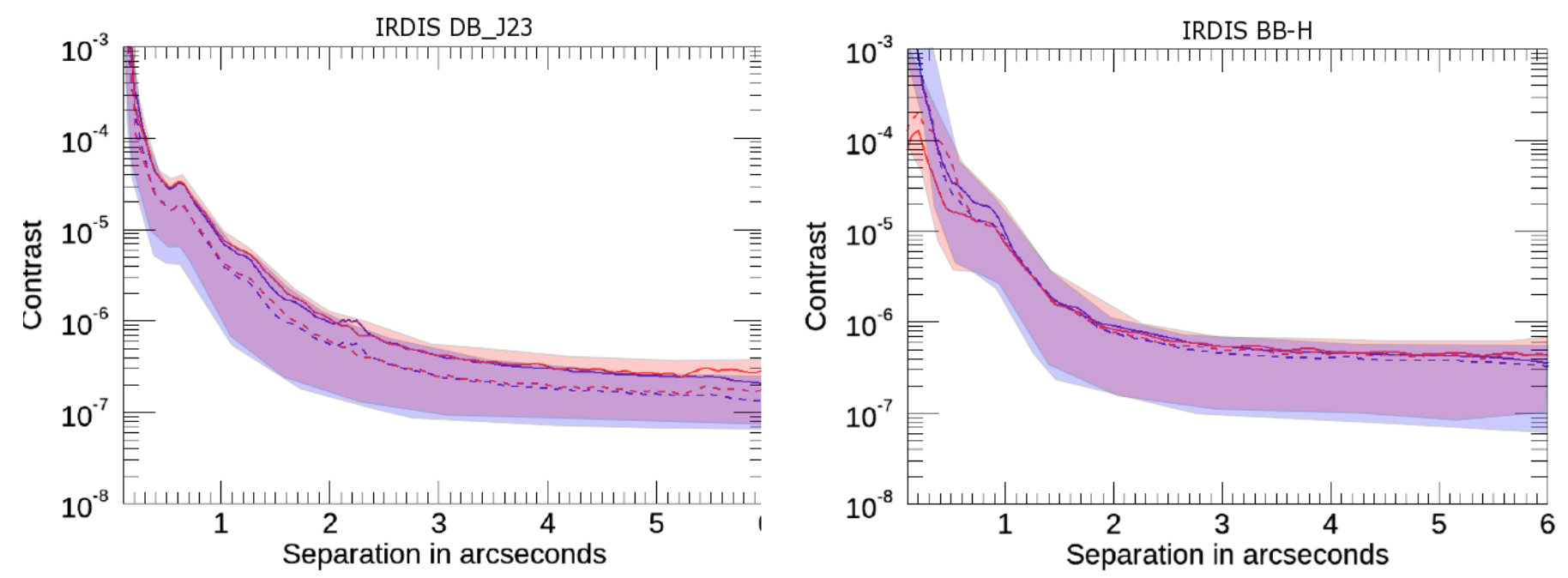

Fig. 5. Contrast curves at $5 \sigma$ obtained for a small subsample of targets dedicated to follow up for IRDIS mode observations. The solid line gives the median value of the contrast. The dashed line gives the mean value of the contrast. Red indicates IRDIS data reduced in PCA ADI; blue indicates IRDIS data reduced in TLOCI ADI; and green indicates IFS with a PCA ASDI reduction.
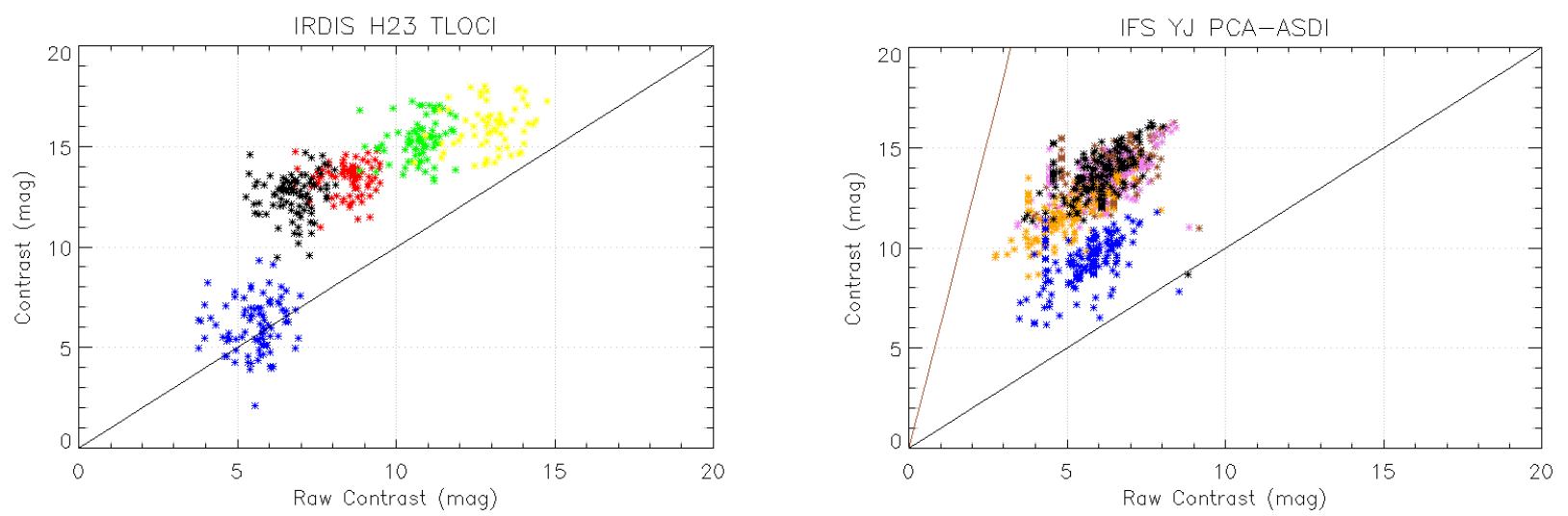

Fig. 6. IRDIS and IFS processed contrast computed using respectively TLOCI and PCA-ASDI as function of raw contrast at various separations in irdifs mode. Blue is for 100 mas separation, orange for 200 mas separation and pink for 400 mas separations, black for 500 mas separation, brown for 700 mas separation, red for 1000 mas separation, green for 2000 mas separation and yellow for 4000 mas separations. The black and brown lines represent, respectively, a gain of 1 and 6.25 , assuming 39 spectral channels. In fact, there are 15 independent spectral IFS channels leading to a maximum gain of 3.9 when neglecting the possible combination of the two IRDIS channels.

is performed using TLOCI. On the contrary, the PCA method for both IRDIS and IFS seems to be affected by the magnitude of the star at least for the largest separations, which is likely related to the noise from the instruments' background.

We also highlight that one clear cause of contrast degradation in the post-ADI contrast is the presence of a smooth halo within the AO-corrected region, which results from either bad seeing or from high-altitude wind related to the jet stream as discussed in Cantalloube et al. (2018), leading to a non symmetrical halo in the direction of the wind (called the wind driven halo, WDH). This halo is rotating in the pupil-stabilised data set because it is fixed on the sky as described in Cantalloube et al. (2020). In post-ADI frames, it therefore appears as a brighter elongation along the wind direction, with negative counterparts at 90 degrees. The impact of this effect does not appear to be very strong on the azimuthal averaged contrasts we plotted but it is noticeable on the 2D contrast maps. Increasing the temporal bandwidth of SAXO is the foreseen solution to help mitigate this effect. This is considered as one option in a forthcoming upgrade of the instrument, SPHERE+ (Boccaletti et al. 2020).

\subsection{Mass detection limits}

The contrast curves are converted into mass limits using massluminosity relationships. Whereas for old ( $\gtrsim 1 \mathrm{Gyr}$ ) systems this relationship is essentially unique for gas giants at large separations (Burrows et al. 1997; Baraffe et al. 2003), at young ages, the value of the post-formation luminosity still remains uncertain (Marley et al. 2007; Spiegel \& Burrows 2012; Marleau $\&$ Cumming 2014). We present here a mass conversion of the contrast curves for a few specific targets using the canonical predictions of the COND-2003 evolutionary models (Baraffe et al. 2003). The impact of using other mass-luminosity relationships on the sensitivity of the SHINE survey are explored in more detail in Vigan et al. (2021). For IRDIS contrast curves, we convert the contrast curves using the evolutionary models computed in the appropriate dual-band filter, while for the IFS contrast curves we use the predictions in the $J$-band filter for $\mathrm{YJ}$ data and in the $H$-band filter for YJH data. As demonstrated in Vigan et al. (2015), this approach for the IFS detection limits provides an accurate estimation of the detection limit. 
For a selection of companions presented in Sect. 5.1, we show the detection of planet-like limits achieved by the survey in Fig. 7. Several targets appear to be relatively easy (HR 8799 bcd, HIP 65426, and HIP 64892) for the purposes of this survey, allowing for exquisite characterisation. Other targets appear to be much more challenging mainly because they are at separations closer than $20 \mathrm{AU}$. The detection limits for these typical objects reach few Jupiter masses at such small separations, while it can reach around 1 Jupiter mass at separations greater than 50 AU.

\section{Exoplanet and candidate companion detections}

\subsection{Brown dwarfs and exoplanets}

A total of sixteen substellar companions were imaged over the course of this phase of the SHINE survey, including seven brown dwarf companions (PZ TelB, $\eta$ TelB, CD 352722 B, HIP 78530 B, HIP 107412 B, GSC 8047-0232 B, and HIP $64892 \mathrm{~B}$ ), and ten planetary-mass companions (51 Erib, $\beta$ Pictoris b, HD 95086 b, HR8799 bcde, GJ 504 b, AB Pic b, and HIP 65426 b). Two new companions were discovered within this sample: the exoplanet HIP 65426 b (Chauvin et al. 2017b) and the brown dwarf companion to HIP 64892 B (Cheetham et al. 2018). PDS 70 was not originally part of the SHINE sample and was specifically targeted to explore the properties of the transition disk that led to the discovery of the first planet PDS $70 \mathrm{~b}$ in this system (Keppler et al. 2018; Müller et al. 2018), as well as the confirmation of the second planet PDS 70 c (Mesa et al. 2019). The other substellar companions, known from previous direct imaging campaigns, originally were blindly selected based on their host star properties, but then carefully characterized to study their orbital, spectral and physical properties in connection sometimes with their environment (the presence of additional planets or disk structures in the system). The $\mathrm{S} / \mathrm{N}$ maps for a selection of candidates are shown in Fig. 8 for IFS data and Fig. 9 for IRDIS data. The extracted spectro-photometric data are reported in Fig. 10. The measured position, contrast, semimajor axis, and predicted mass for each companion are listed in Table 1. The detection limits for a few of these systems are shown in Fig. 7 as an illustration of the SPHERE performances. The Sco-Cen members HIP 71724 and HIP 73990, with their stellar to substellar-mass companions previously discovered in sparseaperture masking by Hinkley et al. (2015), were observed, but the close companions were not resolved given their probable small separations at the epoch of our observations.

In addition to the discovery and characterization papers on HIP 65426 b and HIP 64892 B summarized below, in-depth studies of several of these companions, based on from the SHINE sample, have been published in these early years of the survey: PZ Tel B (Maire et al. 2016a), HIP 107412 B (Milli et al. 2017a; Delorme et al. 2017b) for the brown dwarf companion, and HR 8799 b,c,d,e (Zurlo et al. 2016; Bonnefoy et al. 2016), 51 Erib (Samland et al. 2017; Maire et al. 2019), HD 95086 b (Chauvin et al. 2018), $\beta$ Pictoris b (Lagrange et al. 2019), and GJ 504 b (Bonnefoy et al. 2018) for the imaged exoplanets.

\subsubsection{HIP 65426 b}

HIP $65426 \mathrm{~b}$ was the first planet discovered with SPHERE as part of the SHINE Survey (Chauvin et al. (2017b)). This warm, dusty giant planet is orbiting at a relatively large projected angular distance of 830 mas (92 au projected) from its intermediatemass primary (Figs. 8 and 9). Multi-epoch observations confirm that it shares common proper motion with HIP 65426, a young
A2 member of the $17 \mathrm{Myr}$ old Lower Centaurus-Crux association. Spectro-photometric measurements extracted with IFS and IRDIS, as shown in Fig. 10, between 0.95 and $2.2 \mu \mathrm{m}$, indicate a warm, dusty atmosphere characteristics of young low-surface gravity L5-L7 dwarfs. Hot-start evolutionary models predict a luminosity consistent with a 6-12 $M_{\text {Jup }}, T_{\text {eff }}=1300-1600 \mathrm{~K}$ and $R=1.5 R_{\text {Jup }}$ for the planet. These results were later confirmed by Cheetham et al. (2019) combining SPHERE and $\mathrm{NaCo}$ observations.

Given its physical and spectral properties, HIP 65426 b represents a particularly interesting case for studying the presence of clouds as a function of particle size, composition, and location in the atmosphere, in order to search for signatures of non-equilibrium chemistry, and, finally, to test models of planet formation and evolution (Cheetham et al. 2018). The planet location would not favor a formation by core accretion unless HIP 65426 b formed significantly closer to the star followed by a planet-planet scattering event. Dedicated simulations by Marleau et al. (2019) show that core formation at small separations from the star followed by outward scattering and runaway accretion at a few hundred astronomical units succeeds in reproducing the mass and separation of HIP 65426 b. In such a scenario, the planet is predicted to have a high eccentricity $(\geq 0.5)$ and to be accompanied by one or several roughly Jovian-mass planets at smaller semi-major axes, which also could have a high eccentricity. SHINE detection limits setting relatively good constraints at close separation ( $2 M_{\text {Jup }}$ beyond $20 \mathrm{au}$ ), as shown in Fig. 7, do not exclude the presence of unseen inner massive planets in that system that could have scattered out HIP 65426 b.

\subsubsection{HIP 64892 B}

A bright brown dwarf companion was also discovered around the star HIP 64892, which is a B9.5V member of the Lower Centaurus-Crux association. The measured angular separation of the companion (about 1.27") corresponds to a projected distance of $159 \mathrm{au}$. We observed this target with IRDIS dual-band imaging and in long-slit spectroscopy to estimate its spectral energy distribution and astrometry. More details can be found in the discovery paper by Cheetham et al. (2018). The luminosity and spectrum are consistent with a young (16 Myr) brown dwarf of low surface gravity and with a spectral type M9. From comparison with the BT-Settl atmospheric models its effective temperature was estimated to $T_{\text {eff }}=2600 \mathrm{~K}$ and a comparison of the companion photometry to the COND evolutionary models yields a mass between 29 and $37 M_{\text {Jup }}$. In spite of this, HIP 64892 is one of the highest mass stars around which a substellar companion has been detected due to the challenges associated with observing such stars and the tendency for large surveys to focus on solar-like or low-mass stars. HIP 64892 is a rare example of high or intermediate mass stars with extreme-mass ratio $(q=0.01)$ companions at large separations and will be useful for testing models relating to the formation and evolution of such low-mass objects.

\subsection{Colors and spectra of detected substellar companions}

Figure 10 shows the spectra of the brown dwarf and exoplanet companions detected within the IFS field of view: 51 Eri b, $\beta$ Pic b, HD 95086 b, HIP 65426 b, HR 8799 d and e, HIP 107412 B and PZ Tel B. Most of these spectra are very red and can be classified as of L- or early T-type. The only middle T-type object detected in the IFS field of view is 51 Eri b (Samland et al. 2017), while $\mathrm{PZ}$ Tel B is an M-type object (Maire et al. 2016a). These very 

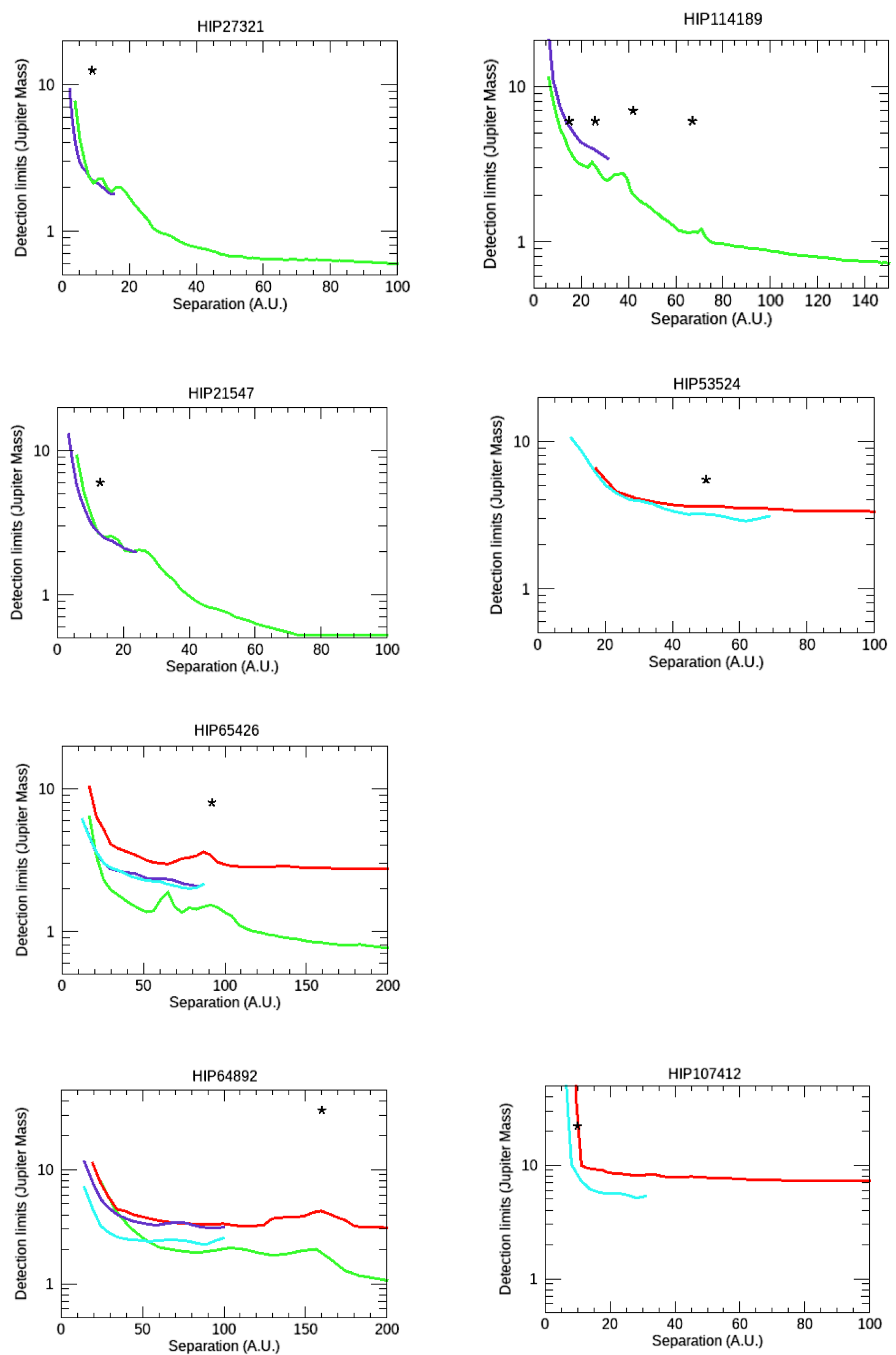

Fig. 7. SPHERE IRDIS and IFS mass detection limits. The IFS H data are represented in blue, the IFS YJ data are represented in violet, while the IRDIS H23 and IRDIS K12 data are represented respectively in green and red. The limits have been converted into mass using the COND-2003 evolutionary models (Baraffe et al. 2003). 

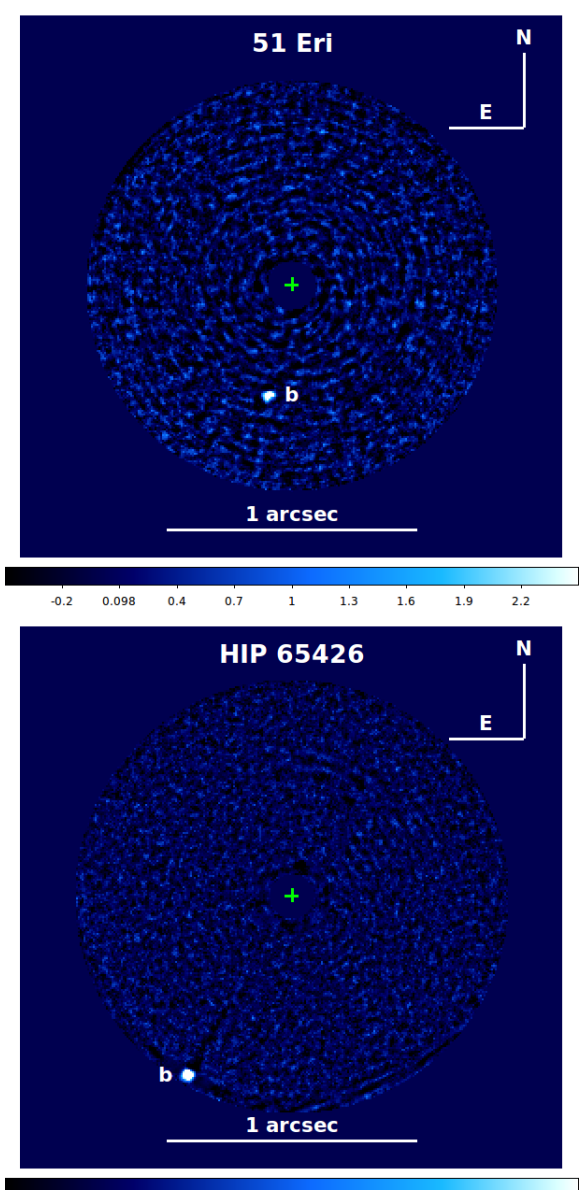

$\begin{array}{cccc}-0.4 & 0.2 & 0.8 & 1.4\end{array}$
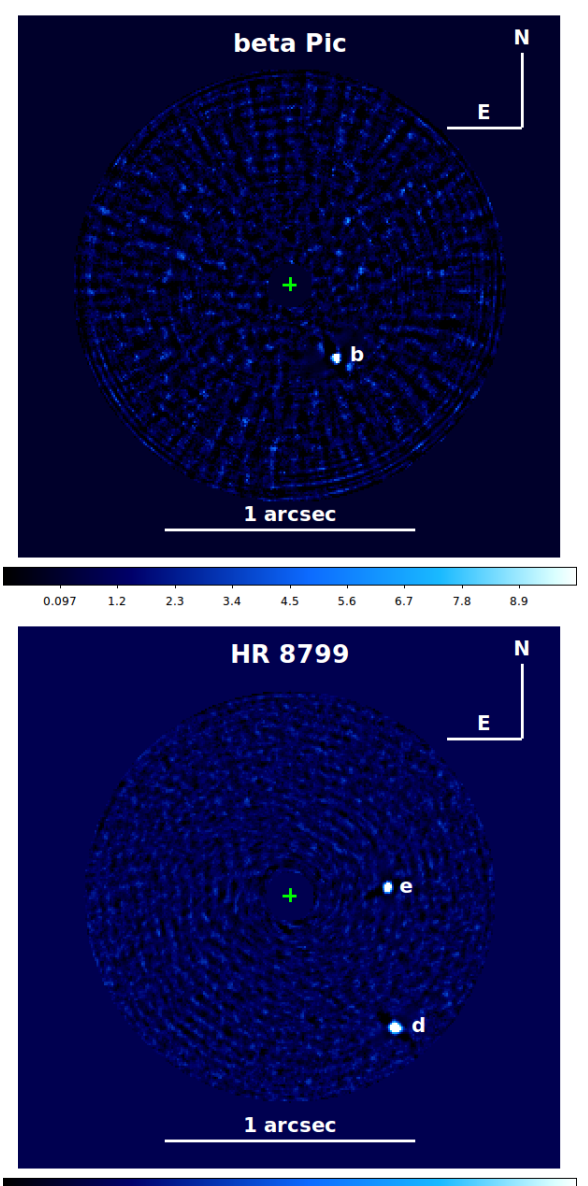

$\begin{array}{cccc}-0.4 & 0.2 & 0.8 & 1.4\end{array}$
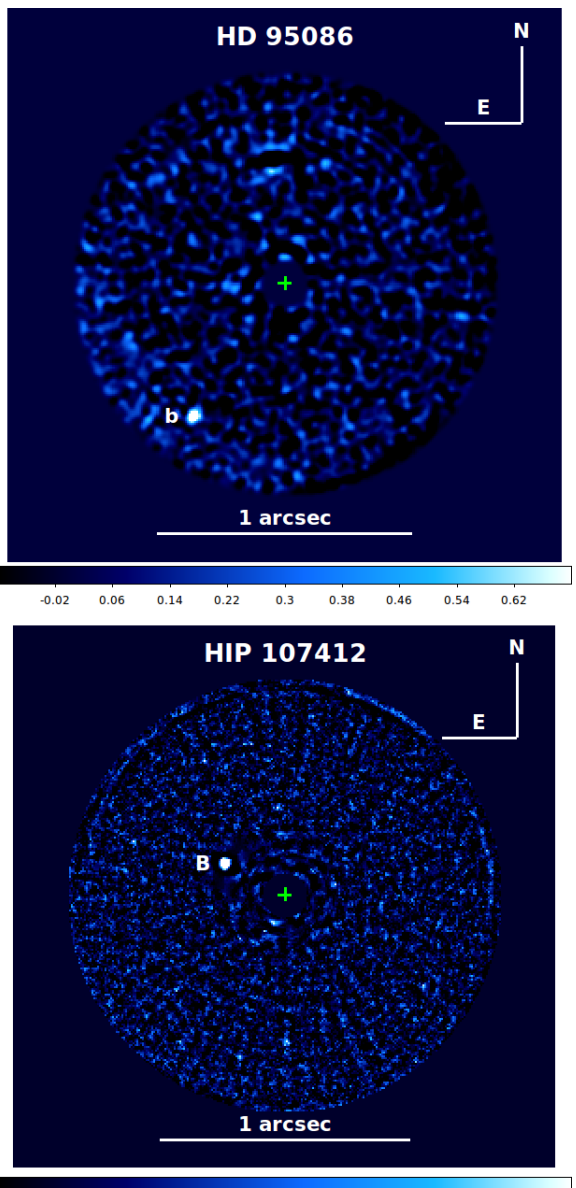

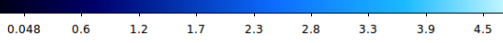

Fig. 8. S/N maps for the sample data where at least one substellar close companion was detected with IFS. In each panel, the green cross marks the star's position.

red colors were quite unexpected for such faint objects, indicating that young substellar objects have a spectrum that is quite different from those of older ones of the same luminosity, which can be interpreted as an indication for the presence of dust in their atmosphere (Chauvin et al. 2004; Bonnefoy et al. 2014). From the point of view of planet detection - the focus of this paper - the implication is that young substellar companions may be below the detection limit in the $Y$ - and $J$-band, and even faint in the $H$-band, while they may be detected quite easily in the $K$ band (extension of the datacubes to short wavelengths is however useful to model and better subtract speckles). An extreme case of this behavior is represented by HD $95086 \mathrm{~b}$ (Chauvin et al. 2018). This has a significant impact on the detection limits of our survey and points to the need for model atmospheres that include dust in order to properly derive the respective masses from the observed magnitudes.

\subsection{Ranking and identification of SHINE candidates}

We identified 1483 unique candidates in the IRDIS images (all epochs), 1176 of which were re-detected at several epochs or using archival data and 307 of which were only detected at a single epoch. Most of the candidates have been observed with the H23 filters (>95\%). Sixty-nine candidates have K1-K2 photometry extracted from the IRDIFS_EXT observations. They were identified in follow-up observations of known systems (HD 95086, HIP 107412, HIP 65426, HIP 64892, CD-35 2722) or, for the most part, in first-epoch observations of stars belonging to the Sco-Cen association for which the detection of late-L dusty companions with red near-infrared colors is favored at $K$-band (e.g., HD 95086b; Chauvin et al. 2018).

We used color-magnitude diagrams (CMDs) to identify the most promising candidates and to perform an efficient follow-up campaign. The candidate absolute magnitudes were computed assuming they are bound and at the same distance as the observed star. Most contaminants are expected to be M- and Ktype stars (e.g., Parravano et al. 2011). Their characteristic colors as well as the range of contrasts probed by the observations creates a locus which can fall into a distinctive color-luminosity space from the sequence of substellar objects in the CMDs. The diagrams are described in detail in Bonnefoy et al. (2018) and are enriched by the recent photometry and distances of reference objects measured by Müller et al. (2018), Mesa et al. (2019), and Dupuy et al. (2020).

The high number of candidates identified in the $\mathrm{H} 23$ observations allows for the building of a well-defined locus of contaminants in the corresponding CMD (see Fig. 11). The locus shows an elongation along a vector that may be related to the distribution of spectral type of the contaminants combined to interstellar extinction (the fainter the object with respect to the host star, the higher the optical extinction and color reddening). The locus intersects the sequence of substellar objects at the L/T transition and, therefore, it cannot discriminate contaminants from bound companions falling in that luminosity range. We could, however, 

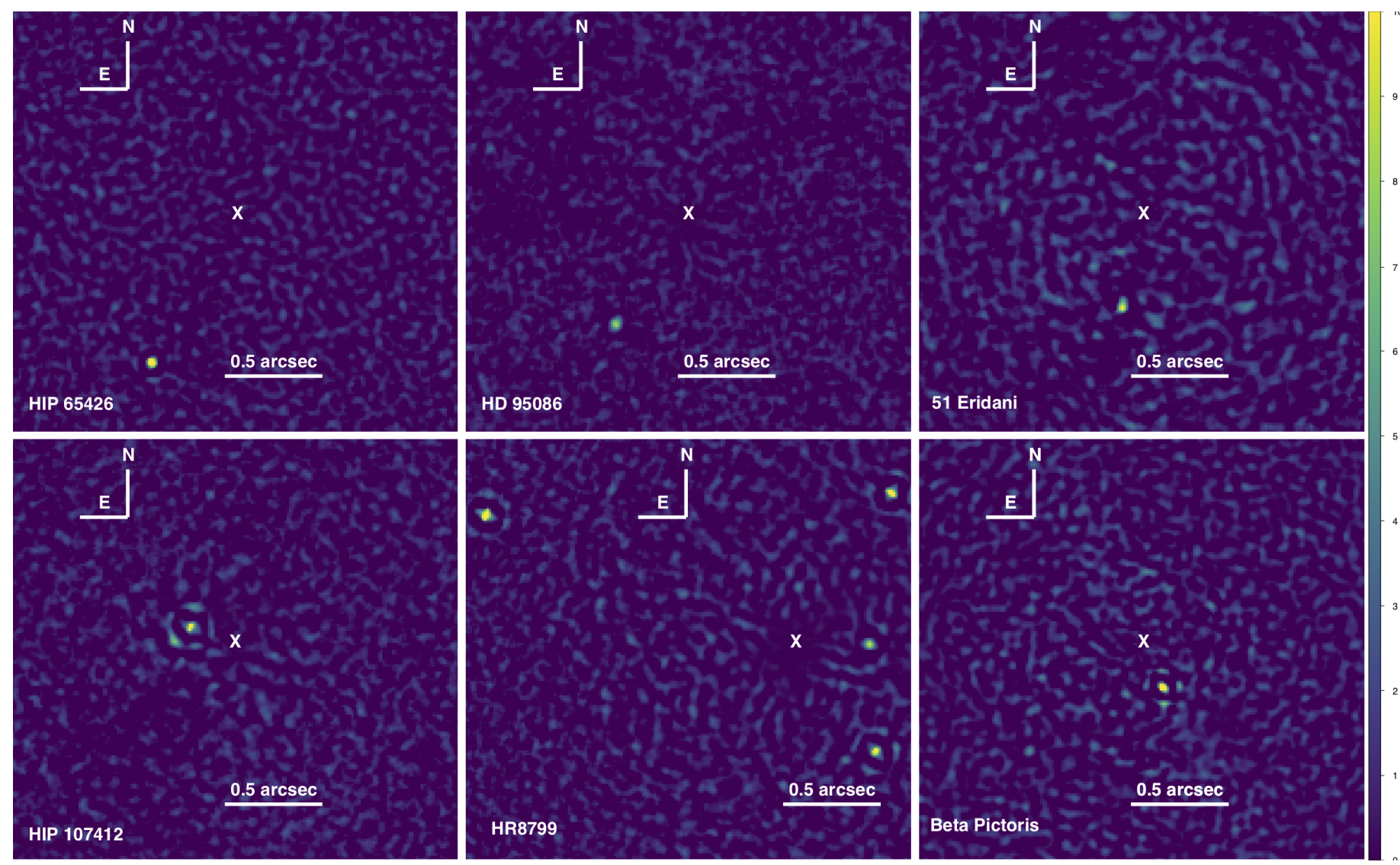

Fig. 9. S/N maps for the sample data where at least one substellar close companion was detected with IRDIS. In each panel, the white cross marks the star's position.

define an exclusion zone (grey shaded region) where substellar companions are not expected to produce such colors and absolute magnitudes. The zone is defined to avoid missing (1) young mid- and late- T-type planets such as 51 Eri b (Macintosh et al. 2015) which can have reddened colors with respect to mature field T-dwarfs; and (2) young planets at the L/T transition such as HR 8799, which can appear under-luminous with respect to older objects in the same temperature range. This criterion was used to identify $44 \%$ of the candidates with $\mathrm{H} 23$ photometry as likely contaminants for which a re-observation was not warranted. Consequently, we decided to prioritize the observations of candidates falling right on the sequence of late-M to mid-L dwarfs and for which the locus spans a narrow range of bluer colors in the same luminosity range. This allowed us to identify HIP 64892B and HIP 65426b as promising candidates before they could be confirmed as co-moving objects. We could also identify blindly known companions (e.g., HIP 78530B, $\eta$ Tel B, PZ Tel B, AB Pic b, CD-35 2722B, HIP 73990B) as promising candidates.

Most of the remaining ambiguous candidates were observed at a second epoch to determine whether they are co-moving companions. Two crowded fields were observed with the $J 23$ filter set. As shown in Bonnefoy et al. (2018), the locus of contaminants falls at a distinct location from the substellar objects in these CMDs and allowed us to classify most of these candidates as contaminants. The locus in the $\mathrm{K} 1 \mathrm{~K} 2$ diagrams is more dispersed and the error bars on the photometry tend to be wider owing to the higher thermal background at these wavelengths. Therefore, detections in $\mathrm{K} 1 \mathrm{~K} 2$ require follow-up observations to clarify the nature of the detections.
The final status of the candidates is given in Table A.1. The companions with a confirmed common-proper motion were flagged as "C". The contaminants either discriminated using the CMD or the common-proper motion test were flagged as "B". Ambiguous cases were flagged as "A". In addition, we report several non-straightforward, non-clear detections ("NC") in that table, which correspond to the identification of candidates with unreliable astrometry or photometry and for which our standard classification scheme could not be applied.

\section{Disks detected in SHINE}

While it is not specifically optimized for this purpose, our observations allow for the detection of circumstellar disks thanks to the exquisite sensitivity and large FoV of IRDIS, twelve SHINE targets, out of which three were new detections. By the construction of our survey, they are debris disk bodies, in fact, we did not consider stars with gas-rich disks in order to reduce the concern related to the impact of disks in planet detection. Table 2 presents a compilation of the main data for the disks we detected; a gallery of their appearance from IFS and IRDIS data are presented in Fig. 12 and Fig. 13 respectively. Detailed analyses of each one of these disks is the subject of specific studies, most of them already having been published (see Table 2).

Not surprisingly, stars with detected debris disks are typically young; the median age is 24 Myr (data from Paper I) and there is only one target with an age older than $50 \mathrm{Myr}$ (HIP $682=$ HD 377). This result is similar to that obtained by the survey by Esposito et al. (2020). We notice that most of the detected disks are seen at a high inclination, $i$, with a median 

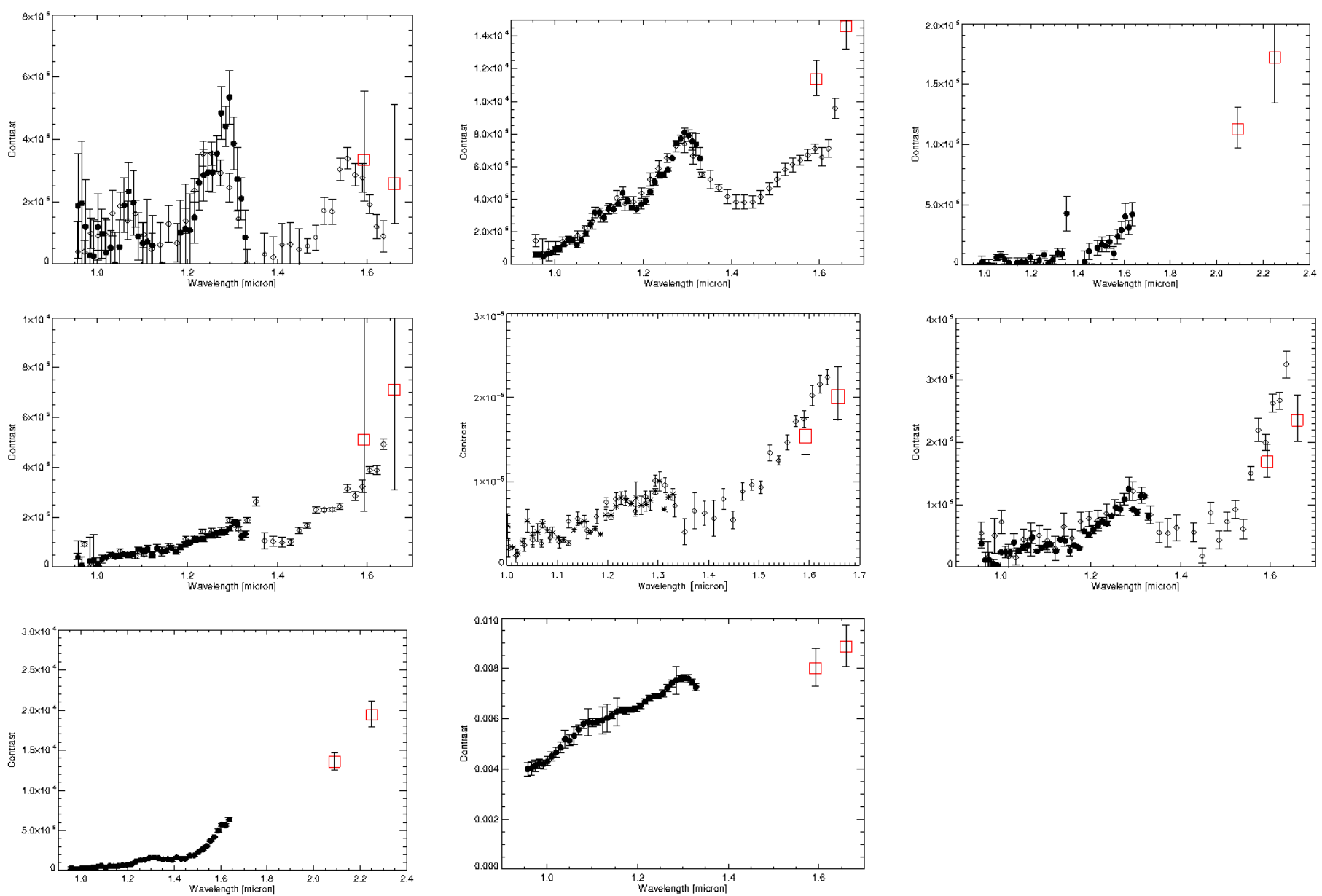

Fig. 10. SPHERE contrast spectra of substellar companions detected within the sample of stars considered in this paper, shown as black symbols Upper row: left: 51 Eri b; center: $\beta$ Pic b; right: HD 95086 b. Middle row: left: HIP 65426 b; center: HR 8799 d; right: HR 8799 e. Lower row: left: HIP 107412 B; center: PZ Tel B. Results shown are obtained by making a median of results at different epochs with good quality results. Spectra obtained with the $Y J$ and $Y H$ mode were kept separate. The error bar is either the result of the scatter of results at different epochs, or if only one was available in that epoch, the error bar obtained by considering other positions at the same separation from the star. The spectra shown were obtained using ASDI PCA and corrected for the attenuation factor using fake planets at similar separation. This method is reasonable when the contrast is very large, so that the planet cannot be seen well at individual wavelengths. Red symbols are the contrast values obtained from IRDIS data. We note that there is some offset between IFS and IRDIS contrasts. This is at least in part due to the non-optimal extraction of IFS spectra shown in this figure. More accurate spectral extractions may be found in papers discussing the individual targets.
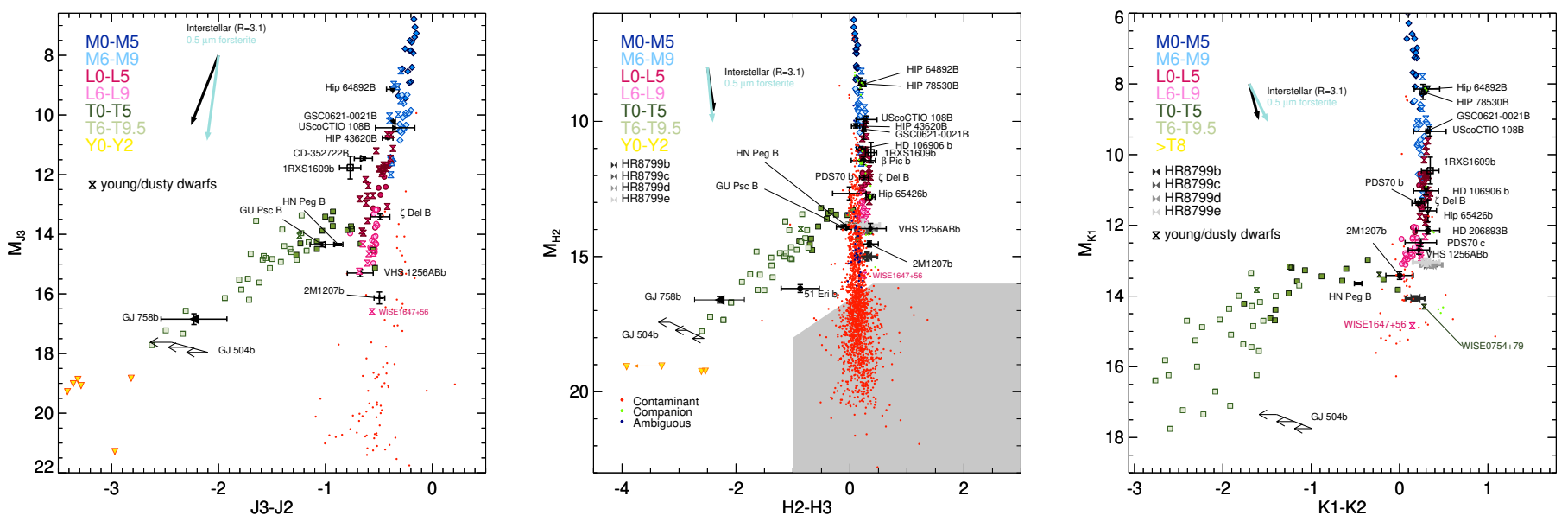

Fig. 11. Photometry of the candidates (spots) observed in the sample of 159 stars and reported in color-magnitude diagrams, assuming a common distance with the host-star. The grey zone represents the excluded background-like point sources. 
Table 2. Debris disk detected from the SHINE survey (F150 sample).

\begin{tabular}{lccccc}
\hline \hline Star & SpT & $\begin{array}{c}M_{\mathrm{G}} \\
\text { mag }\end{array}$ & $\begin{array}{c}\text { Age } \\
\text { Myr }\end{array}$ & $\begin{array}{c}i \\
\text { Degree }\end{array}$ & References \\
\hline \multicolumn{5}{c}{ New SHINE detections } \\
\hline HIP 73145 & A2IV & 7.87 & 17 & 72.6 & Feldt et al. (2017) \\
HIP 86598 / HD 160305 & F8/G0V & 8.18 & 24 & 82.0 & Perrot, Clément et al. (2019) \\
HIP 59960 / HD 106906 & F5V & 7.68 & 13 & 5 & Lagrange et al. (2016) \\
\hline \multicolumn{7}{c}{ Previously } & known disks & \\
\hline P Pic b & A6V & 3.72 & 24 & $>89$ & Lagrange et al. (2019) \\
AU Mic & M1V & 7.84 & 24 & $>89$ & Boccaletti et al. (2018) \\
HIP 682/ HD 377 & G2V & 7.44 & 150 & & Langlois et al. (in prep.) \\
HIP 11360 / HD 15115 & F4IV & 6.68 & 45 & 85.8 & Engler et al. (2019) \\
HIP 36948 / HD 61005 & G8V & 8.00 & 50 & 84.5 & Olofsson et al. (2016) \\
TWA 7 & M2V & 10.62 & 10 & 13 & Olofsson et al. (2018) \\
TWA 11 / HR4796 & A0V & 5.77 & 10 & 76.4 & Milli et al. (2017c) \\
HIP 64995 / HD 115600 & F2IV/V & 8.14 & 16 & 80 & Gibbs et al. (2019) \\
NZ Lup & G2 & 7.78 & 16 & 86 & Boccaletti et al. (2019) \\
\hline
\end{tabular}

value of $i=82$ degree. The only disk that has a low inclination in our survey (TWA 7) was detected only in polarimetry Olofsson et al. (2018). The strong dependence on inclination is due to the combination of a deeper optical depth (debris disks being optically thin), of higher efficiency of forward scattering, and of the use of angular differential imaging to achieve a deeper contrast (see Milli et al. 2012; Sissa et al. 2018). This selection effect would be less severe using polarimetric observations, as is often done when studying disks (see the case of TWA 7; see also Esposito et al. 2020). The strong selection bias towards edge-on disks implies that debris disks at the typical age of our targets should be much more common than indicated by eleven detections over a sample of 150 stars. If we indeed consider only the 52 stars with age $<30$ Myr in our sample, we detected a disk for around 8 of them. In five cases, the inclination is $i>80$ degrees, an occurrence that has a $17 \%$ probability of happening. This suggests that debris disks that are similar to the ones we detected surround a large fraction of young stars (see Sissa et al. 2018 for a similar argument about the M-stars in our survey or Meshkat et al. 2017).

Debris disks have a limited impact on the detection limits of point sources. Low-inclination debris disks have negligible effects because they are optically thin and well below the detection limit. In what concerns high inclination disks, the effect is more relevant (see Milli et al. 2012 for a discussion). They typically cover a small fraction of the sky area; however, planetary orbit is possibly coplanar with the disk, so that the sky region covered by the disk is most likely where there are planets. As discussed by Milli et al. (2012), the flux loss depends on the technique used, and may be large for some of them. On the other hand, in addition to flux losses, Milli et al. (2012) showed that disk features may lead to false positive point sources detections. Furthermore, the multi-wavelength approach allowed by simultaneously using both IFS and IRDIS provides more diagnostics for an appropriate classification of detected features and, more particularly, point sources. The good sensitivity of ASDIPCA to structures within disks is clearly shown by the case of AU Mic, where this technique allows to detect very faint pointlike sources with contrasts of $\sim 14.5 \mathrm{mag}$ at $S / N>10$ along the edge-on disk (Boccaletti et al. 2018). The S/N of these detections agree with the expectations, based on our estimate of the contrast limit at this separation based on the whole images.
We note that these structures are, nonetheless, not planets: the spectrum is flat, inconsistent with that of young planets, and the objects are far too bright to be reflecting planets. We carefully considered a similar possibility for all our targets and we are confident that there is no false positive among our detections.

Concerning the detection of the disk structures themselves, the standard ADI methods used in this paper lead to several known causes of artifacts altering both the morphology and the photometry of the disks. In particular they suffer from either positive or negative replicas and from the self-subtraction phenomenon which can only be alleviated by using a physical model of instrumental effects as recently proposed by Pairet et al. (2021); Flasseur et al. (2020c). However, these are beyond the scope of this paper.

\section{Conclusions and prospects}

We process, in a uniform manner, more than 300 datasets from the SPHERE/SHINE Survey obtained at the VLT/ESO in visitor mode and assess the SHINE survey's typical sensitivity as a function of the host star and observing conditions. From these first 150 stars observed out of the planned 400-star survey, we achieved typical contrasts of $10^{6}$ at $1^{\prime \prime}$ separation and reached up to a $10^{7}$ contrast at $2^{\prime \prime}$ separation for the best observations. SPHERE's SHINE delivers one of the few largest and deepest direct imaging surveys for exoplanets conducted to date. Compared to GPIES, SHINE has been operating in priority visitor mode and its observing conditions in regular visitor mode are highly variable and generally not as good, leading to a wider spreading of the contrast performances.

Sixteen substellar companions around twelve host stars have been detected within the first half of the SHINE survey: eight brown dwarfs and eight planetary-mass companions. This paper summarizes the measured position, contrast, and derived mass for each companion and provides astro-photometric data for all the candidates detections. The two new discoveries in the first half of the SHINE survey are: the planetary mass companion to HIP $65426 \mathrm{~b}$ (Chauvin et al. 2017b) and the brown dwarf companion to HIP 64892 B (Cheetham et al. 2018). All other substellar companions characterized during SHINE were discovered in previous direct imaging campaigns. 

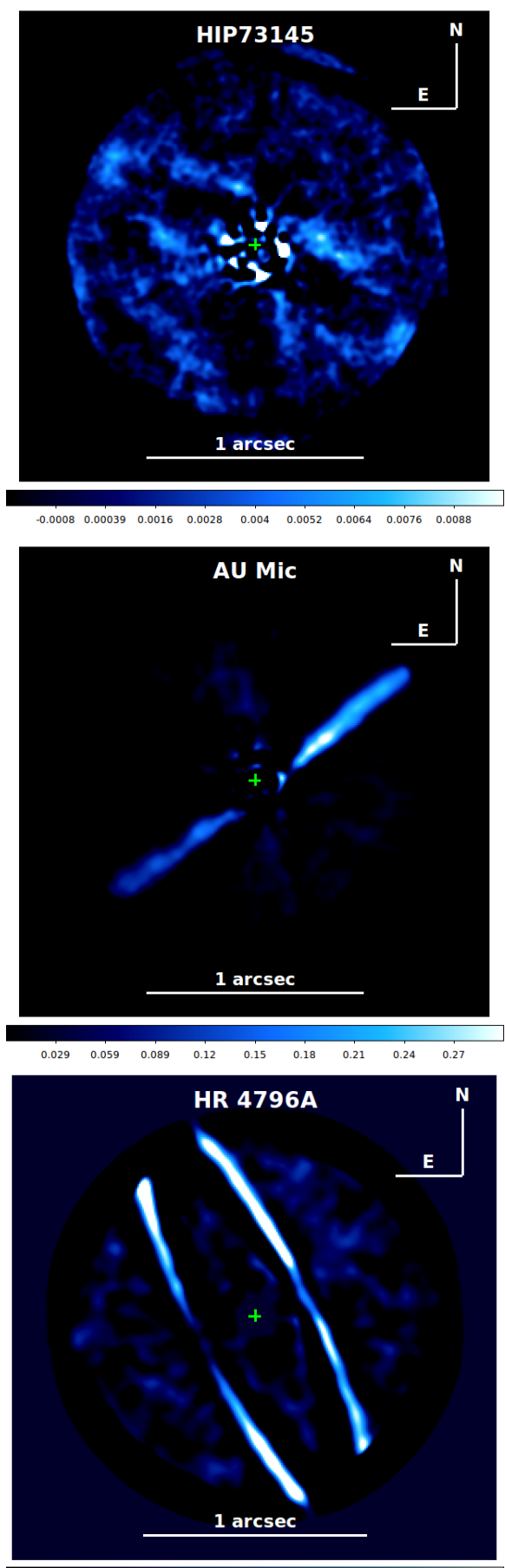

$\begin{array}{llllllllll}0.0005 & 0.0060 & 0.0115 & 0.0170 & 0.0225 & 0.0280 & 0.0335 & 0.0390 & 0.0445\end{array}$
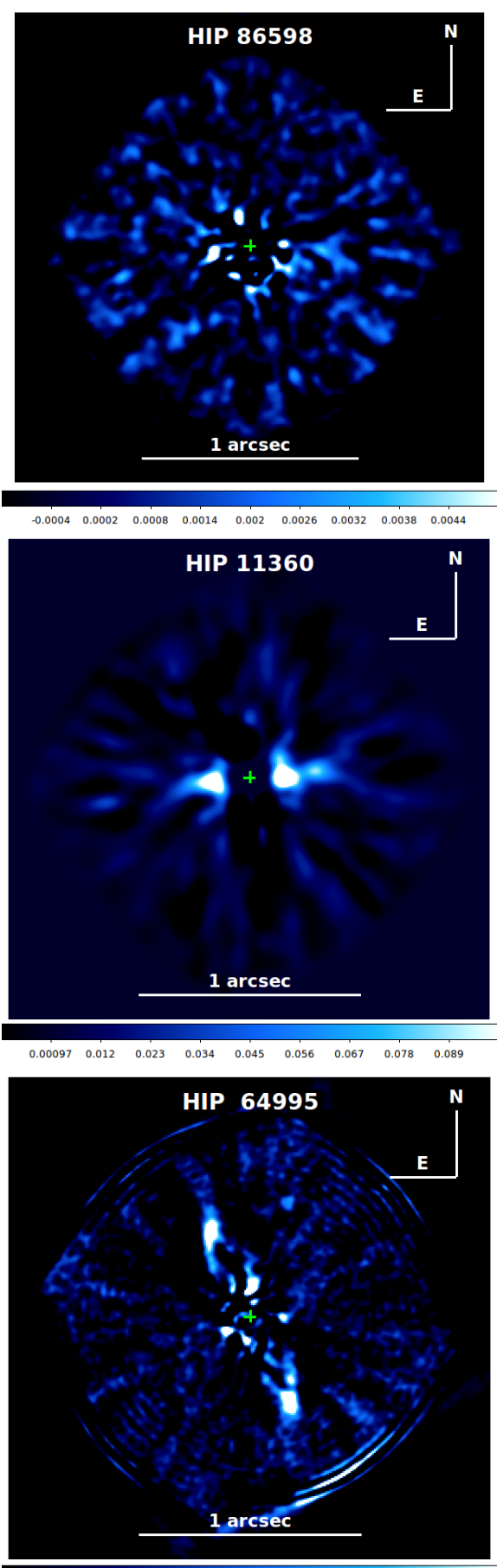

$\begin{array}{lllllllll}9.7-05 & 0.0012 & 0.0023 & 0.0034 & 0.0045 & 0.0056 & 0.0067 & 0.0078 & 0.0089\end{array}$
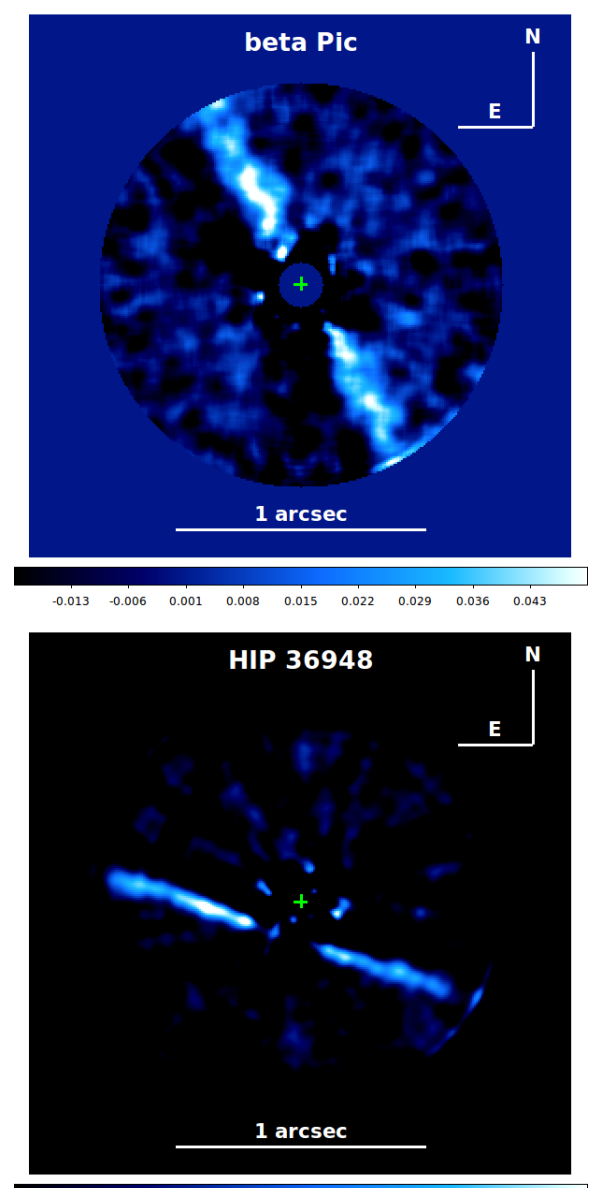

$\begin{array}{llllllllll}0.0021 & 0.0052 & 0.0083 & 0.0114 & 0.0145 & 0.0176 & 0.0207 & 0.0238 & 0.0269\end{array}$

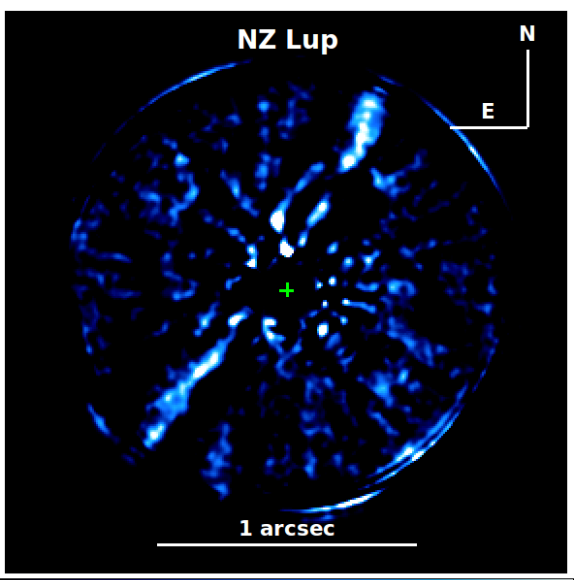

0.00100

Fig. 12. Gallery of images of debris disks in the F100 sample detected with IFS. Some detections are rather marginal (e.g., HIP 86598). The disk is not detectable for TWA7 and HIP 682 (it was, however, detected with SPHERE using other approaches). Whenever possible, we combined several images to have a cleaner detection. This is the reason the planet is not visible in the beta Pic image. Generally, we used ASDI PCA with 25 modes, which is a quite conservative method, and smoothed the images to have a better view. In each panel, the green cross marks the star's position.

The SHINE survey is due to be completed in 2021, but it will certainly extend over several more years to become complete in terms of follow-ups for all candidates within at least a 300 au and possibly even farther away. The final sample will include over 500 stars, which will make SHINE the largest high-contrast imaging survey to date, covering from B to M stars in the solar neighborhood. The reanalysis of the complete SHINE data with advanced processing techniques (Cantalloube et al. 2015; Ruffio et al. 2017; Flasseur et al. 2018, 2020b,a; Berdeu et al. 2020) will be performed in order to provide improved detection limits and reach the full power of this survey with the use of the full sample.

There are a limited number of bright, young, nearby stars available to high-contrast direct imagers like SPHERE and GPI. As a result, at the current achievable contrasts, we cannot expect a significant number of new detections of imaged giant planets following the completion of both GPIES and SPHERE/SHINE (Desidera et al. 2021) campaigns. An improvement in performance from instrument upgrades (Boccaletti et al. 2020, 

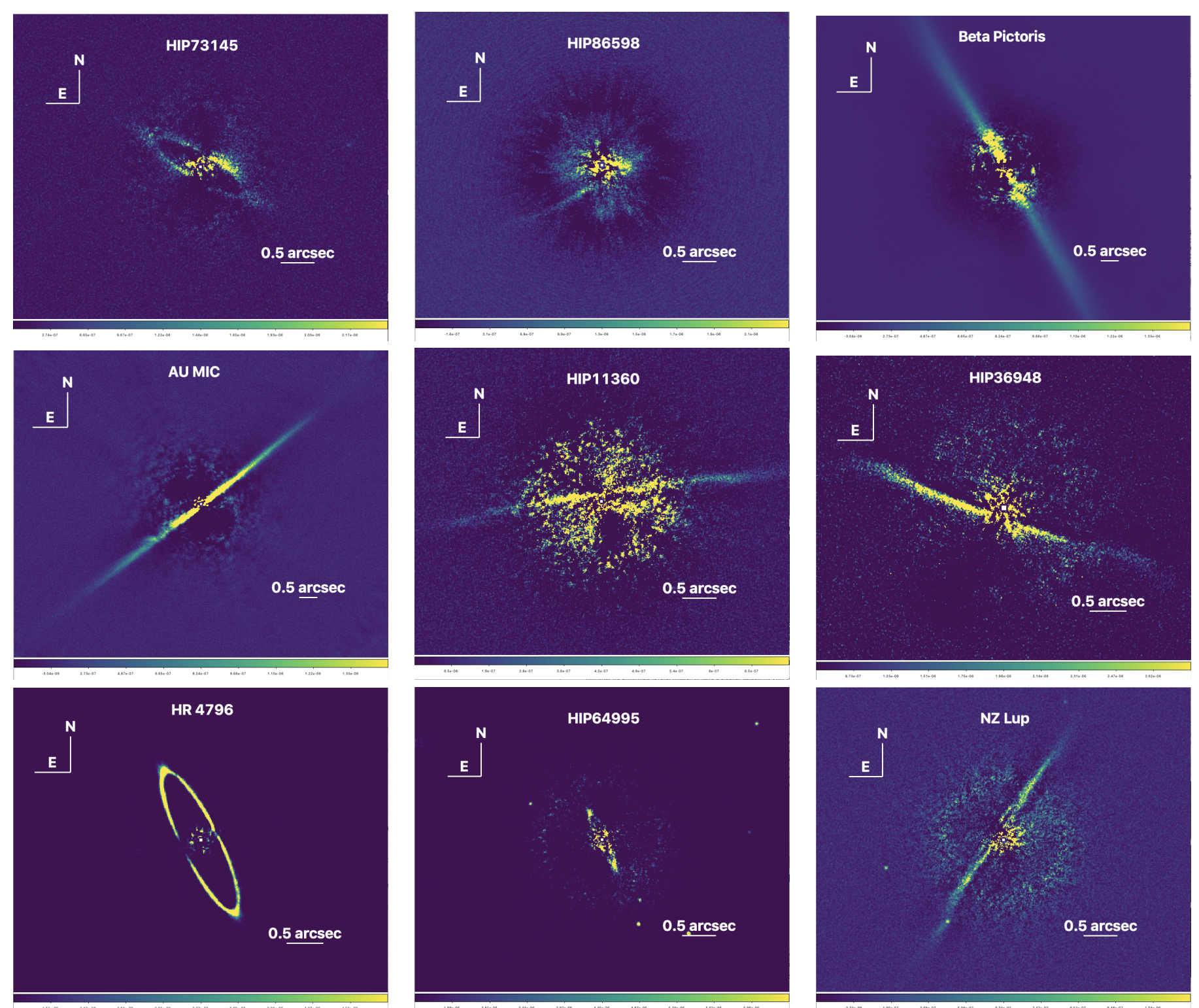

Fig. 13. Gallery of images of debris disks in the F100 sample detected with IRDIS obtained using PCA with five modes in $\mathrm{H}_{2}$. Some of the detections are marginal, as they are better detected with other signal extraction approaches.

Chilcote et al. 2018), however, could unlock mass and separation phase spaces around these target stars that are currently inaccessible, as these are also likely to host more planets Wagner et al. (2019). Additional planets discovered with upgraded GPI and SPHERE would allow us to test the robustness of the trends with stellar mass and planetary mass uncovered in this paper. The Gaia mission will identify astrometric signatures of planets that may be confirmed with direct imaging, which can also boost the number of companions in this separation range. Finally, cross checks with results from other indirect techniques, for instance, transits or high-precision radial velocity, will allow us to achieve a much better picture of the architecture of planetary systems.

Acknowledgements. SPHERE is an instrument designed and built by a consortium consisting of IPAG (Grenoble, France), MPIA (Heidelberg, Germany), LAM (Marseille, France), LESIA (Paris, France), Laboratoire Lagrange (Nice, France), INAF - Osservatorio di Padova (Italy), Observatoire de Genève (Switzerland), ETH Zürich (Switzerland), NOVA (Netherlands), ONERA (France) and ASTRON (Netherlands) in collaboration with ESO. SPHERE was funded by ESO, with additional contributions from CNRS (France), MPIA
(Germany), INAF (Italy), FINES (Switzerland) and NOVA (Netherlands). SPHERE also received funding from the European Commission Sixth and Seventh Framework Programmes as part of the Optical Infrared Coordination Network for Astronomy (OPTICON) under grant number RII3-Ct-2004-001566 for FP6 (2004-2008), grant number 226604 for FP7 (2009-2012) and grant number 312430 for FP7 (2013-2016). This paper is based on observations collected at the European Southern Observatory under ESO programmes 198.C0209, 097.C-0865, 095.C-0298, 095.C-0309,096.C-0241. This work has made use of the SPHERE Data Centre, jointly operated by OSUG/IPAG (Grenoble), PYTHEAS/LAM/CeSAM (Marseille), OCA/Lagrange (Nice), Observatoire de Paris/LESIA (Paris), and Observatoire de Lyon (OSUL/CRAL). This work is supported by the French National Research Agency in the framework of the Investissements d'Avenir program (ANR-15-IDEX-02), through the funding of the "Origin of Life" project of the Univ. Grenoble-Alpes. This work is jointly supported by the French National Programms (PNP and PNPS) and by the Action Spécifique Haute Résolution Angulaire (ASHRA) of CNRS/INSU co-funded by CNES. We also thank the anonymous referee for her/his careful reading of the manuscript as well as her/his insightful comments and suggestions. AV acknowledges funding from the European Research Council (ERC) under the European Union's Horizon 2020 research and innovation programme (grant agreement No. 757561). A.-M.L. acknowledges funding from French National Research Agency (GIPSE project). C.P. acknowledges financial support from Fondecyt (grant 3190691) and financial support from the ICM (Iniciativa Científica 
Milenio) via the Núcleo Milenio de Formación Planetaria grant, from the Universidad de Valparaíso. T.H. acknowledges support from the European Research Council under the Horizon 2020 Framework Program via the ERC Advanced Grant Origins 832428 .

\section{References}

Baraffe, I., Chabrier, G., Barman, T. S., Allard, F., \& Hauschildt, P. H. 2003, A\&A, 402, 701

Bellini, A., Anderson, J., \& Bedin, L. R. 2011, PASP, 123, 622

Bellini, A., Anderson, J., van der Marel, R. P., et al. 2014, ApJ, 797, 115

Bellini, A., Bianchini, P., Varri, A. L., et al. 2017, ApJ, 844, 167

Berdeu, A., Soulez, F., Denis, L., Langlois, M., \& Thiébaut, É. 2020, A\&A, 635, A90

Beuzit, J. L., Vigan, A., Mouillet, D., et al. 2019, A\&A, 631, A155

Biller, B. A., Close, L. M., Masciadri, E., et al. 2007, ApJS, 173, 143

Biller, B. A., Liu, M. C., Wahhaj, Z., et al. 2010, ApJ, 720, L82

Biller, B. A., Liu, M. C., Wahhaj, Z., et al. 2013, ApJ, 777, 160

Boccaletti, A., Carbillet, M., Fusco, T., et al. 2008, SPIE Conf. Ser., 7015, 70156E

Boccaletti, A., Sezestre, E., Lagrange, A. M., et al. 2018, A\&A, 614, A52

Boccaletti, A., Thébault, P., Pawellek, N., et al. 2019, A\&A, 625, A21

Boccaletti, A., Chauvin, G., Mouillet, D., et al. 2020, ArXiv e-prints [arXiv:2003.05714]

Bohn, A. J., Kenworthy, M. A., Ginski, C., et al. 2020, ApJ, 898, L16

Bonnefoy, M., Chauvin, G., Lagrange, A. M., et al. 2014, A\&A, 562, A127

Bonnefoy, M., Zurlo, A., Baudino, J. L., et al. 2016, A\&A, 587, A58

Bonnefoy, M., Perraut, K., Lagrange, A. M., et al. 2018, A\&A, 618, A63

Boss, A. P. 1997, Lunar Planet. Sci. Conf., 28, 137

Bowler, B. P., Liu, M. C., Shkolnik, E. L., \& Tamura, M. 2015, ApJS, 216, 7

Burrows, A., Marley, M., Hubbard, W. B., et al. 1997, ApJ, 491, 856

Cantalloube, F., Mouillet, D., Mugnier, L. M., et al. 2015, A\&A, 582, A89

Cantalloube, F., Por, E. H., Dohlen, K., et al. 2018, A\&A, 620, L10

Cantalloube, F., Farley, O. J. D., Milli, J., et al. 2020, A\&A, 638, A98

Carbillet, M., Bendjoya, P., Abe, L., et al. 2011, Exp. Astron., 30, 39

Chauvin, G., Thomson, M., Dumas, C., et al. 2003, A\&A, 404, 157

Chauvin, G., Lagrange, A. M., Dumas, C., et al. 2004, A\&A, 425, L29

Chauvin, G., Lagrange, A. M., Lacombe, F., et al. 2005a, A\&A, 430, 1027

Chauvin, G., Lagrange, A. M., Zuckerman, B., et al. 2005b, A\&A, 438, L29

Chauvin, G., Lagrange, A. M., Bonavita, M., et al. 2010, A\&A, 509, A52

Chauvin, G., Faherty, J., Boccaletti, A., et al. 2012, A\&A, 548, A33

Chauvin, G., Desidera, S., Lagrange, A. M., et al. 2017a, in SF2A-2017: Proceedings of the Annual meeting of the French Society of Astronomy and Astrophysics, ed. C. Reylé, P. Di Matteo, F. Herpin, E. Lagadec, A. Lançon, Z. Meliani, \& F. Royer, Di

Chauvin, G., Desidera, S., Lagrange, A. M., et al. 2017b, A\&A, 605, L9

Chauvin, G., Gratton, R., Bonnefoy, M., et al. 2018, A\&A, 617, A76

Cheetham, A., Bonnefoy, M., Desidera, S., et al. 2018, A\&A, 615, A160

Cheetham, A. C., Samland, M., Brems, S. S., et al. 2019, A\&A, 622, A80

Chilcote, J. K., Bailey, V. P., De Rosa, R., et al. 2018, Proc. SPIE Conf. Ser., 10702, 1070244

Claudi, R. U., Turatto, M., Gratton, R. G., et al. 2008, SPIE Conf. Ser., 7014, 70143E

Close, L. M., Puglisi, A., Males, J. R., et al. 2012, ApJ, 749, 180

Close, L. M., Males, J. R., Morzinski, K., et al. 2013, ApJ, 774, 94

Cumming, A., Butler, R. P., Marcy, G. W., et al. 2008, PASP, 120, 531

Delorme, P., Lagrange, A. M., Chauvin, G., et al. 2012, A\&A, 539, A72

Delorme, P., Meunier, N., Albert, D., et al. 2017a, in SF2A-2017: Proceedings of the Annual meeting of the French Society of Astronomy and Astrophysics, Di

Delorme, P., Schmidt, T., Bonnefoy, M., et al. 2017b, A\&A, 608, A79

De Rosa, R. J., Rameau, J., Patience, J., et al. 2016, ApJ, 824, 121

De Rosa, R. J., Nguyen, M. M., Chilcote, J., et al. 2020, J. Astron. Teles. Instrum., Syst., 6, 015006

Desidera, S., Chauvin, G., Bonavita, M., et al. 2021, A\&A, 651, A70

Dohlen, K., Langlois, M., Saisse, M., et al. 2008, SPIE Conf. Ser., 7014, 70143L

Dupuy, T. J., Liu, M. C., Magnier, E. A., et al. 2020, Res. Notes Am. Astron. Soc., 4, 54

Ehrenreich, D., Lagrange, A. M., Montagnier, G., et al. 2010, A\&A, 523, A73

Engler, N., Boccaletti, A., Schmid, H. M., et al. 2019, A\&A, 622, A192

Esposito, T. M., Kalas, P., Fitzgerald, M. P., et al. 2020, AJ, 160, 24

Feldt, M., Olofsson, J., Boccaletti, A., et al. 2017, A\&A, 601, A7

Flasseur, O., Denis, L., Thiébaut, É., \& Langlois, M. 2018, A\&A, 618, A138

Flasseur, O., Denis, L., Thiébaut, É., \& Langlois, M. 2020a, A\&A, 637, A9

Flasseur, O., Denis, L., Thiébaut, É., \& Langlois, M. 2020b, A\&A, 634, A2

Flasseur, O., Denis, L., Thiébaut, E., \& Langlois, M. 2020c, A\&A, submitted

Fusco, T., Rousset, G., Sauvage, J. F., et al. 2006, Opt. Exp., 14, 7515

Fusco, T., Sauvage, J.-F., Petit, C., et al. 2014, Proc. SPIE, 9148, 1
Fusco, T., Sauvage, J.-F., Mouillet, D., et al. 2016, Proc. SPIE, 9909, 99090U Gaia Collaboration (Brown, A. G. A., et al.) 2018, A\&A, 616, A1

Galicher, R., \& Marois, C. 2011, in Second International Conference on Adaptive Optics for Extremely Large Telescopes. Online at http: //ao4elt2 . lesia. obspm. fr, P25

Galicher, R., Boccaletti, A., Mesa, D., et al. 2018, A\&A, 615, A92

Gibbs, A., Wagner, K., Apai, D., et al. 2019, AJ, 157, 39

Guerri, G., Daban, J.-B., Robbe-Dubois, S., et al. 2011, Exp. Astron., 30, 59

Haffert, S. Y., Por, E. H., Keller, C. U., et al. 2020, A\&A, 635, A56

Harayama, Y., Eisenhauer, F., \& Martins, F. 2008, ApJ, 675, 1319

Hennebelle, P., \& Chabrier, G. 2011, ApJ, 743, L29

Hinkley, S., Bowler, B. P., Vigan, A., et al. 2015, ApJ, 805, L10

Janson, M., Bonavita, M., Klahr, H., et al. 2011, ApJ, 736, 89

Jovanovic, N., Guyon, O., Lozi, J., et al. 2016, Proc. SPIE Conf. Ser., 9909 99090W

Kasper, M., Apai, D., Janson, M., \& Brandner, W. 2007, A\&A, 472, 321

Keppler, M., Benisty, M., Müller, A., et al. 2018, A\&A, 617, A44

Khorrami, Z., Lanz, T., Vakili, F., et al. 2016, A\&A, 588, L7

Konopacky, Q. M., Rameau, J., Duchêne, G., et al. 2016, ApJ, 829, L4

Kuzuhara, M., Tamura, M., Kudo, T., et al. 2013, ApJ, 774, 11

Lafrenière, D., Doyon, R., Marois, C., et al. 2007, ApJ, 670, 1367

Lafrenière, D., Jayawardhana, R., Janson, M., et al. 2011, ApJ, 730, 42

Lagrange, A. M., Gratadour, D., Chauvin, G., et al. 2009, A\&A, 493, L21

Lagrange, A.-M., Rubini, P., Brauner-Vettier, N., et al. 2016, Proc. SPIE, 9910, 991033

Lagrange, A. M., Boccaletti, A., Langlois, M., et al. 2019, A\&A, 621, L8

Lannier, J., Delorme, P., Lagrange, A. M., et al. 2016, A\&A, 596, A83

Lowrance, P. J., Schneider, G., Kirkpatrick, J. D., et al. 2000, ApJ, 541, 390

Macintosh, B., Graham, J. R., Barman, T., et al. 2015, Science, 350, 64

Maire, A. L., Bonnefoy, M., Ginski, C., et al. 2016a, A\&A, 587, A56

Maire, A.-L., Langlois, M., Dohlen, K., et al. 2016b, SPIE Conf. Ser., 9908, 990834

Maire, A. L., Rodet, L., Cantalloube, F., et al. 2019, A\&A, 624, A118

Makidon, R. B., Sivaramakrishnan, A., Perrin, M. D., et al. 2005, PASP, 117, 831

Marleau, G.-D., \& Cumming, A. 2014, MNRAS, 437, 1378

Marleau, G.-D., Coleman, G. A. L., Leleu, A., \& Mordasini, C. 2019, A\&A, 624, A20

Marley, M. S., Fortney, J. J., Hubickyj, O., Bodenheimer, P., \& Lissauer, J. J. 2007, ApJ, 655, 541

Marois, C., Lafrenière, D., Doyon, R., Macintosh, B., \& Nadeau, D. 2006, ApJ, 641,556

Marois, C., Macintosh, B., Barman, T., et al. 2008, Science, 322, 1348

Marois, C., Zuckerman, B., Konopacky, Q. M., Macintosh, B., \& Barman, T. 2010, Nature, 468, 1080

Marois, C., Correia, C., Véran, J.-P., \& Currie, T. 2014, IAU Symp., 299, 48

Masciadri, E., Mundt, R., Henning, T., Alvarez, C., \& Barrado y Navascués, D. 2005, ApJ, 625, 1004

Mawet, D., Milli, J., Wahhaj, Z., et al. 2014, ApJ, 792, 97

Mesa, D., Gratton, R., Zurlo, A., et al. 2015, A\&A, 576, A121

Mesa, D., Keppler, M., Cantalloube, F., et al. 2019, A\&A, 632, A25

Meshkat, T., Mawet, D., Bryan, M. L., et al. 2017, AJ, 154, 245

Milli, J., Mouillet, D., Lagrange, A. M., et al. 2012, A\&A, 545, A111

Milli, J., Hibon, P., Christiaens, V., et al. 2017a, A\&A, 597, L2

Milli, J., Mouillet, D., Fusco, T., et al. 2017b, AO4ELT5 Proc., Adaptive Optics for Extremely Large Telescopes 5, Conf. Proc. June 25-30, Tenerife, Canary Islands, Spain

Milli, J., Vigan, A., Mouillet, D., et al. 2017c, A\&A, 599, A108

Mugrauer, M., Vogt, N., Neuhäuser, R., \& Schmidt, T. O. B. 2010, A\&A, 523, L1

Müller, A., Keppler, M., Henning, T., et al. 2018, A\&A, 617, L2

Nakajima, T., Oppenheimer, B. R., Kulkarni, S. R., et al. 1995, Nature, 378 463

Nielsen, E. L., Liu, M. C., Wahhaj, Z., et al. 2013, ApJ, 776, 4

Nielsen, E. L., De Rosa, R. J., Macintosh, B., et al. 2019, AJ, 158, 13

Olofsson, J., Samland, M., Avenhaus, H., et al. 2016, A\&A, 591, A108

Olofsson, J., van Holstein, R. G., Boccaletti, A., et al. 2018, A\&A, 617, A109

Pairet, B., Cantalloube, F., \& Jacques, L. 2021, MNRAS, 503, 3724

Parravano, A., McKee, C. F., \& Hollenbach, D. J. 2011, ApJ, 726, 27

Pavlov, A., Möller-Nilsson, O., Feldt, M., et al. 2008, Proc. SPIE Confe. Ser. 7019, 701939

Perrot, Clément, Thebault, Philippe, Lagrange, Anne-Marie, et al. 2019, A\&A, 626, A95

Petit, C., Sauvage, J.-F., Sevin, A., et al. 2012, Proc. SPIE, 8447, 844717

Rameau, J., Chauvin, G., Lagrange, A. M., et al. 2013a, ApJ, 772, L15

Rameau, J., Chauvin, G., Lagrange, A. M., et al. 2013b, A\&A, 553, A60

Rochau, B., Brandner, W., Stolte, A., et al. 2010, ApJ, 716, L90 
Ruffio, J.-B., Macintosh, B., Wang, J. J., et al. 2017, ApJ, 842, 14

Samland, M., Mollière, P., Bonnefoy, M., et al. 2017, A\&A, 603, A57

Sauvage, J.-F., Fusco, T., Petit, C., et al. 2016, J. Astron. Teles. Instrum. Syst., 2, 025003

Sissa, E., Olofsson, J., Vigan, A., et al. 2018, A\&A, 613, L6

Skrutskie, M. F., Cutri, R. M., Stiening, R., et al. 2006, AJ, 131, 1163

Soto, M., Bellini, A., Anderson, J., et al. 2017, AJ, 153, 19

Soummer, R., Pueyo, L., \& Larkin, J. 2012, ApJ, 755, L28

Spiegel, D. S., \& Burrows, A. 2012, ApJ, 745, 174

Vigan, A., Moutou, C., Langlois, M., et al. 2010, MNRAS, 407, 71

Vigan, A., Gry, C., Salter, G., et al. 2015, MNRAS, 454, 129

Vigan, A., Fontanive, C., Meyer, M., et al. 2021, A\&A, 651, A72

Wagner, K., Apai, D., \& Kratter, K. M. 2019, ApJ, 877, 46

Wagner, K., Apai, D., Kasper, M., et al. 2020, ApJ, 902, L6

Wahhaj, Z., Liu, M. C., Biller, B. A., et al. 2011, ApJ, 729, 139

Wang, J., Mawet, D., Fortney, J. J., et al. 2018, AJ, 156, 272

Zurlo, A., Vigan, A., Mesa, D., et al. 2014, A\&A, 572, A85

Zurlo, A., Vigan, A., Galicher, R., et al. 2016, A\&A, 587, A57

${ }^{1}$ CRAL, UMR 5574, CNRS, Université de Lyon, ENS, 9 avenue Charles André, 69561 Saint Genis Laval Cedex, France e-mail: maud.langlois@univ-lyon1.fr

2 Aix Marseille Univ, CNRS, CNES, LAM, Marseille, France

${ }^{3}$ INAF - Osservatorio Astronomico di Padova, Vicolo della Osservatorio 5, 35122 Padova, Italy

${ }^{4}$ Univ. Grenoble Alpes, CNRS, IPAG, 38000 Grenoble, France

${ }^{5}$ LESIA, Observatoire de Paris, Université PSL, CNRS, Sorbonne Université, Univ. Paris Diderot, Sorbonne Paris Cité, 5 place Jules Janssen, 92195 Meudon, France

${ }^{6}$ Max Planck Institute for Astronomy, Königstuhl 17, 69117 Heidelberg, Germany

7 STAR Institute, Université de Liège, Allée du Six Août 19c, 4000 Liège, Belgium

${ }^{8}$ Geneva Observatory, University of Geneva, Chemin des Mailettes 51, 1290 Versoix, Switzerland

9 Department of Astronomy, University of Michigan, Ann Arbor, MI 48109, USA

${ }^{10}$ Institute for Particle Physics and Astrophysics, ETH Zurich, Wolfgang-Pauli-Strasse 27, 8093 Zurich, Switzerland

11 Institute for Astronomy, University of Edinburgh, EH9 3HJ, Edinburgh, UK
${ }^{12}$ Scottish Universities Physics Alliance (SUPA), Institute for Astronomy, University of Edinburgh, Blackford Hill, Edinburgh EH9 3HJ, UK

${ }^{13}$ Center for Space and Habitability, University of Bern, 3012 Bern, Switzerland

${ }^{14}$ Université Côte d'Azur, OCA, CNRS, Lagrange, France

15 European Southern Observatory, Karl-Schwarzschild-Str. 2, 85748 Garching, Germany

${ }^{16}$ European Southern Observatory, Alonso de Cordova 3107, Casilla 19001 Vitacura, Santiago 19, Chile

${ }^{17}$ Núcleo de Astronomía, Facultad de Ingeniería y Ciencias, Universidad Diego Portales, Av. Ejercito 441, Santiago, Chile

18 Escuela de Ingeniería Industrial, Facultad de Ingeniería y Ciencias, Universidad Diego Portales, Av. Ejercito 441, Santiago, Chile

19 Anton Pannekoek Institute for Astronomy, Science Park 9, 1098 XH Amsterdam, The Netherlands

20 Unidad Mixta Internacional Franco-Chilena de Astronomía, CNRS/INSU UMI 3386 and Departamento de Astronomía, Universidad de Chile, Casilla 36-D, Santiago, Chile

${ }^{21}$ DOTA, ONERA (Office National d'Etudes et de Recherches Arospatiales), Université Paris Saclay, 92322 Chatillon, France

${ }^{22}$ NOVA Optical Infrared Instrumentation Group, Oude Hoogeveensedijk 4, 7991 PD Dwingeloo, The Netherlands

${ }^{23}$ INAF - Osservatorio Astronomico di Capodimonte, Salita Moiariello 16, 80131 Napoli, Italy

${ }^{24}$ Instituto de Física y Astronomía, Facultad de Ciencias, Universidad de Valparaíso, Av. Gran Bretaña 1111, Valparaíso, Chile

25 Núcleo Milenio Formación Planetaria - NPF, Universidad de Valparaíso, Av. Gran Bretaña 1111, Valparaíso, Chile

26 Univ Lyon, UJM-Saint-Etienne, CNRS, Institut d'Optique Graduate School, Laboratoire Hubert Curien UMR 5516, 42023, SAINTETIENNE, France

27 Dipartimento di Fisica e Astronomia “G. Galilei”, Università di Padova, Via Marzolo, 8, 35121 Padova, Italy

28 Department of Astronomy, Stockholm University, AlbaNova University Center, 10691 Stockholm, Sweden

${ }^{29}$ Division of Medical Image Computing, German Cancer Research Center (DKFZ), 69120 Heidelberg, Germany

${ }^{30}$ Ural Federal University, Yekaterinburg 620002, Russia

31 INAF - Catania Astrophysical Observatory, via S. Sofia 78, 95123 Catania, Italy

32 Pixyl, 5 Avenue du Grand Sablon, 38700 La Tronche, France 
M. Langlois et al.: The SPHERE infrared survey for exoplanets (SHINE). II.

Appendix A: Astrophotometric table of detections

Table A.1. Detected point sources parameters.

\begin{tabular}{|c|c|c|c|c|c|c|c|c|c|c|c|c|c|c|c|c|c|c|c|c|c|}
\hline Main_ID & date & filt & ncc & id & sep(mas) & $\begin{array}{l}\text { sep } \\
\text { error }\end{array}$ & $\mathrm{pa}(\mathrm{deg})$ & $\begin{array}{l}\text { pa } \\
\text { error }\end{array}$ & $\mathrm{dm} 0$ & $\begin{array}{l}\mathrm{dm} 0 \\
\text { error }\end{array}$ & $\mathrm{dm} 1$ & $\begin{array}{l}\mathrm{dm} 1 \\
\text { error }\end{array}$ & absm0 & $\begin{array}{l}\text { absm0 } \\
\text { error }\end{array}$ & absm1 & $\begin{array}{l}\text { absm1 } \\
\text { error }\end{array}$ & snr0 & snr1 & proba & color & status \\
\hline HIP53524 & $05 / 05 / 15$ & K12 & 11 & 0.0 & 618.58 & 9.46 & 148.66 & 1.07 & 12.37 & 0.16 & 11.91 & 0.27 & 14.48 & 0.16 & 14.01 & 0.27 & 9.17 & 4.28 & 1.48 & 0.466 & $\mathrm{C}$ \\
\hline HIP53524 & $05 / 05 / 15$ & K12 & 11 & 1.0 & 2212.95 & 17.41 & 35.14 & 0.41 & 13.02 & 0.12 & 13.13 & 0.21 & 15.13 & 0.13 & 15.24 & 0.21 & 17.28 & 5.81 & 22.24 & -0.111 & B \\
\hline HIP53524 & $05 / 05 / 15$ & K12 & 11 & 2.0 & 2331.48 & 39.49 & 305.16 & 0.8 & 0 & 1.09 & 13.64 & 0.34 & 2.11 & 1.09 & 15.75 & 0.34 & 0 & 3.37 & 29.74 & -13.641 & B \\
\hline HIP53524 & $05 / 05 / 15$ & $\mathrm{~K} 12$ & 11 & 3.0 & 4064.85 & 21.13 & 140.74 & 0.1 & 11.87 & 0.11 & 11.79 & 0.11 & 13.98 & 0.11 & 13.89 & 0.12 & 40.09 & 17.63 & 39.61 & 0.086 & B \\
\hline HIP53524 & 05/05/15 & $\mathrm{K} 12$ & 11 & 4.0 & 4432.96 & 22.47 & 320.19 & 0.07 & 6.35 & 0.11 & 6.26 & 0.1 & 8.45 & 0.11 & 8.37 & 0.1 & 88.62 & 105.39 & 1.42 & 0.084 & B \\
\hline HIP53524 & $05 / 05 / 15$ & K12 & 11 & 5.0 & 4669.81 & 23.82 & 246.71 & 0.07 & 11.21 & 0.11 & 11.06 & 0.1 & 13.31 & 0.11 & 13.17 & 0.11 & 43.92 & 29.37 & 37.46 & 0.143 & B \\
\hline HIP53524 & $05 / 05 / 15$ & K12 & 11 & 6.0 & 5024.38 & 28.76 & 244.75 & 0.13 & 13.17 & 0.12 & 13.16 & 0.32 & 15.27 & 0.12 & 15.27 & 0.32 & 18.23 & 3.62 & 74.52 & 0.005 & B \\
\hline HIP53524 & $05 / 05 / 15$ & $\mathrm{~K} 12$ & 11 & 7.0 & 5204.32 & 27.25 & 238.69 & 0.09 & 13.62 & 0.13 & 0 & 1.09 & 15.73 & 0.13 & 2.11 & 1.09 & 16.12 & 0 & 81.89 & 13.619 & B \\
\hline HIP53524 & $05 / 05 / 15$ & $\mathrm{~K} 12$ & 11 & 8.0 & 5585.85 & 28.78 & 322.51 & 0.09 & 12.02 & 0.11 & 11.97 & 0.15 & 14.12 & 0.11 & 14.08 & 0.15 & 31.09 & 9.21 & 63.66 & 0.048 & B \\
\hline HIP53524 & $05 / 05 / 15$ & $\mathrm{~K} 12$ & 11 & 9.0 & 5817.68 & 29.73 & 324 & 0.08 & 11.63 & 0.11 & 11.51 & 0.11 & 13.73 & 0.11 & 13.62 & 0.11 & 35.02 & 18.32 & 59.45 & 0.114 & B \\
\hline HIP53524 & $05 / 05 / 15$ & $\mathrm{~K} 12$ & 11 & 10.0 & 6192.48 & 31.54 & 105.04 & 0.07 & 11.29 & 0.11 & 11.24 & 0.11 & 13.39 & 0.11 & 13.34 & 0.11 & 33.59 & 22.64 & 57.65 & 0.047 & B \\
\hline HIP53524 & $28 / 03 / 18$ & K12 & 11 & 0.0 & 631.9 & 5.65 & 145.52 & 0.63 & 12.27 & 0.08 & 11.83 & 0.14 & 14.37 & 0.08 & 13.93 & 0.14 & 19.91 & 8.82 & 1.46 & 0.439 & $\mathrm{C}$ \\
\hline HIP53524 & $28 / 03 / 18$ & K12 & 11 & 1.0 & 2248.47 & 8.96 & 38.04 & 0.26 & 13.12 & 0.09 & 13.03 & 0.24 & 15.23 & 0.1 & 15.14 & 0.24 & 14 & 4.76 & 23.68 & 0.095 & B \\
\hline HIP53524 & $28 / 03 / 18$ & $\mathrm{~K} 12$ & 11 & 2.0 & 4120.91 & 24.28 & 175.67 & 3.28 & 14.16 & 0.13 & 14.2 & 0.39 & 16.27 & 0.13 & 16.31 & 0.39 & 8.91 & 2.83 & 73.05 & -0.039 & B \\
\hline HIP53524 & $28 / 03 / 18$ & K12 & 11 & 3.0 & 4157.41 & 6.8 & 140.03 & 0.09 & 12.07 & 0.06 & 11.94 & 0.09 & 14.17 & 0.07 & 14.05 & 0.09 & 30.94 & 14.86 & 43.89 & 0.126 & B \\
\hline HIP53524 & $28 / 03 / 18$ & K12 & 11 & 4.0 & 4345.53 & 6.01 & 320.9 & 0.07 & 6.25 & 0.06 & 6.18 & 0.06 & 8.35 & 0.06 & 8.28 & 0.06 & 38.34 & 52.26 & 1.23 & 0.071 & B \\
\hline HIP53524 & $28 / 03 / 18$ & K12 & 11 & 5.0 & 4608.14 & & 245.48 & & 0 & 1.09 & 0 & 1.09 & 2.11 & 1.09 & 2.11 & 1.09 & 0 & 0 & 0.01 & 0 & B \\
\hline HIP53524 & $28 / 03 / 18$ & K12 & 11 & 6.0 & 4953.55 & 12.75 & 243.92 & 0.11 & 13.15 & 0.07 & 13.3 & 0.29 & 15.25 & 0.08 & 15.41 & 0.29 & 20.6 & 3.84 & 73.16 & -0.157 & B \\
\hline HIP53524 & $28 / 03 / 18$ & K12 & 11 & 7.0 & 5135.98 & 15.49 & 237.8 & 0.14 & 13.5 & 0.09 & 13.77 & 0.56 & 15.61 & 0.1 & 15.87 & 0.56 & 13.91 & 1.96 & 79.82 & -0.266 & B \\
\hline HIP53524 & $28 / 03 / 18$ & $\mathrm{~K} 12$ & 11 & 8.0 & 5501.79 & 8.1 & 323.15 & 0.08 & 11.89 & 0.06 & 11.84 & 0.08 & 14 & 0.07 & 13.95 & 0.08 & 31.18 & 22 & 60.31 & 0.054 & B \\
\hline HIP53524 & 28/03/18 & K12 & 11 & 9.0 & 5774.7 & & 325.03 & & 0 & 1.09 & 0 & 1.09 & 2.11 & 1.09 & 2.11 & 1.09 & 0 & 0 & 0.02 & 0 & B \\
\hline HIP53524 & 28/03/18 & $\mathrm{K} 12$ & 11 & 10.0 & 6292.78 & 9.14 & 105.18 & 0.07 & 11.48 & 0.08 & 11.42 & 0.08 & 13.58 & 0.08 & 13.53 & 0.08 & 18.98 & 20.73 & 62.31 & 0.053 & B \\
\hline HIP53524 & $18 / 05 / 19$ & K12 & 9 & 0.0 & 628.43 & 4.76 & 144.11 & 0.53 & 12.21 & 0.14 & 11.72 & 0.13 & 14.32 & 0.14 & 13.83 & 0.13 & 27.33 & 17.6 & 1.4 & 0.492 & $\mathrm{C}$ \\
\hline HIP53524 & $18 / 05 / 19$ & $\mathrm{~K} 12$ & 9 & 1.0 & 2268.61 & 9.16 & 39.36 & 0.26 & 13.08 & 0.14 & 13.29 & 0.23 & 15.19 & 0.14 & 15.4 & 0.23 & 22.04 & 5.25 & 23.72 & -0.21 & B \\
\hline HIP53524 & $18 / 05 / 19$ & K12 & 9 & 2.0 & 4201.34 & 3.81 & 139.74 & 0.08 & 11.97 & 0.14 & 11.82 & 0.12 & 14.08 & 0.14 & 13.92 & 0.12 & 51.51 & 26.64 & 43.16 & 0.155 & B \\
\hline HIP53524 & $18 / 05 / 19$ & $\mathrm{~K} 12$ & 9 & 3.0 & 4305.8 & 2.67 & 321.2 & 0.07 & 6.36 & 0.14 & 6.28 & 0.11 & 8.47 & 0.14 & 8.38 & 0.11 & 51.96 & 80.05 & 1.36 & 0.088 & B \\
\hline HIP53524 & $18 / 05 / 19$ & $\mathrm{~K} 12$ & 9 & 4.0 & 4562.91 & 3.67 & 245.38 & 0.07 & 11.28 & 0.14 & 11.14 & 0.12 & 13.39 & 0.14 & 13.25 & 0.12 & 40.38 & 33.68 & 37.36 & 0.143 & B \\
\hline HIP53524 & $18 / 05 / 19$ & K12 & 9 & 5.0 & 4922.52 & 10.86 & 243.49 & 0.12 & 13.24 & 0.15 & 13.27 & 0.2 & 15.34 & 0.15 & 15.38 & 0.21 & 19.19 & 6.33 & 74.05 & -0.036 & B \\
\hline HIP53524 & $18 / 05 / 19$ & $\mathrm{~K} 12$ & 9 & 6.0 & 5462.93 & 4.69 & 323.43 & 0.08 & 12.01 & 0.14 & 11.96 & 0.12 & 14.12 & 0.14 & 14.07 & 0.12 & 34.68 & 21.48 & 61.93 & 0.052 & B \\
\hline HIP53524 & $18 / 05 / 19$ & $\mathrm{~K} 12$ & 9 & 7.0 & 5697.63 & 4.18 & 324.9 & 0.08 & 11.61 & 0.14 & 11.53 & 0.12 & 13.71 & 0.14 & 13.63 & 0.12 & 35.06 & 28.29 & 57.6 & 0.08 & B \\
\hline HIP53524 & 18/05/19 & K12 & 9 & 8.0 & 6339.33 & 4.79 & 105.24 & 0.07 & 11.43 & 0.14 & 11.35 & 0.12 & 13.53 & 0.15 & 13.45 & 0.12 & 20.34 & 24.84 & 61.93 & 0.082 & B \\
\hline
\end{tabular}

Notes. The full table is available at the CDS. 


\section{Appendix B: Contrast plots}
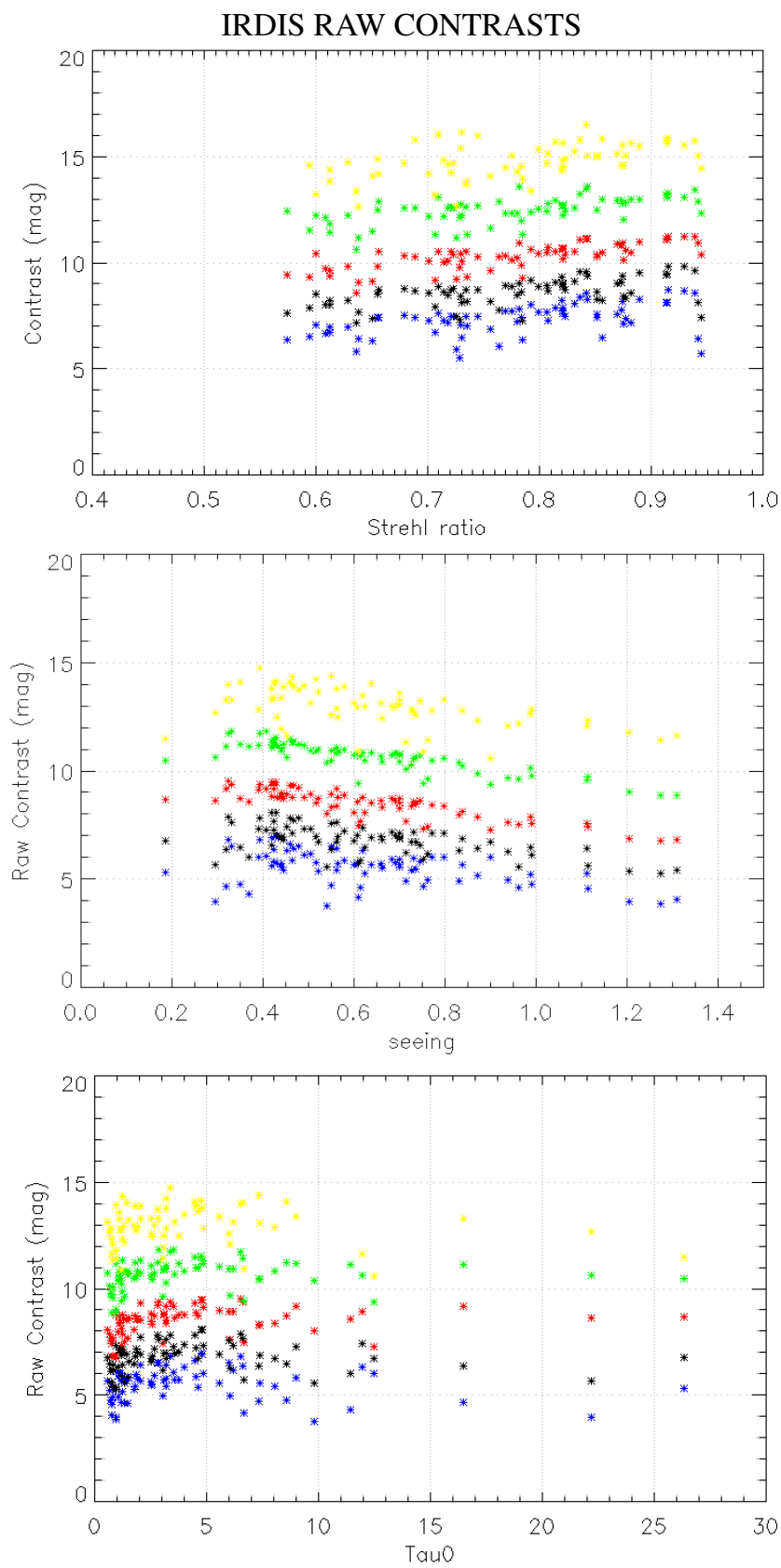
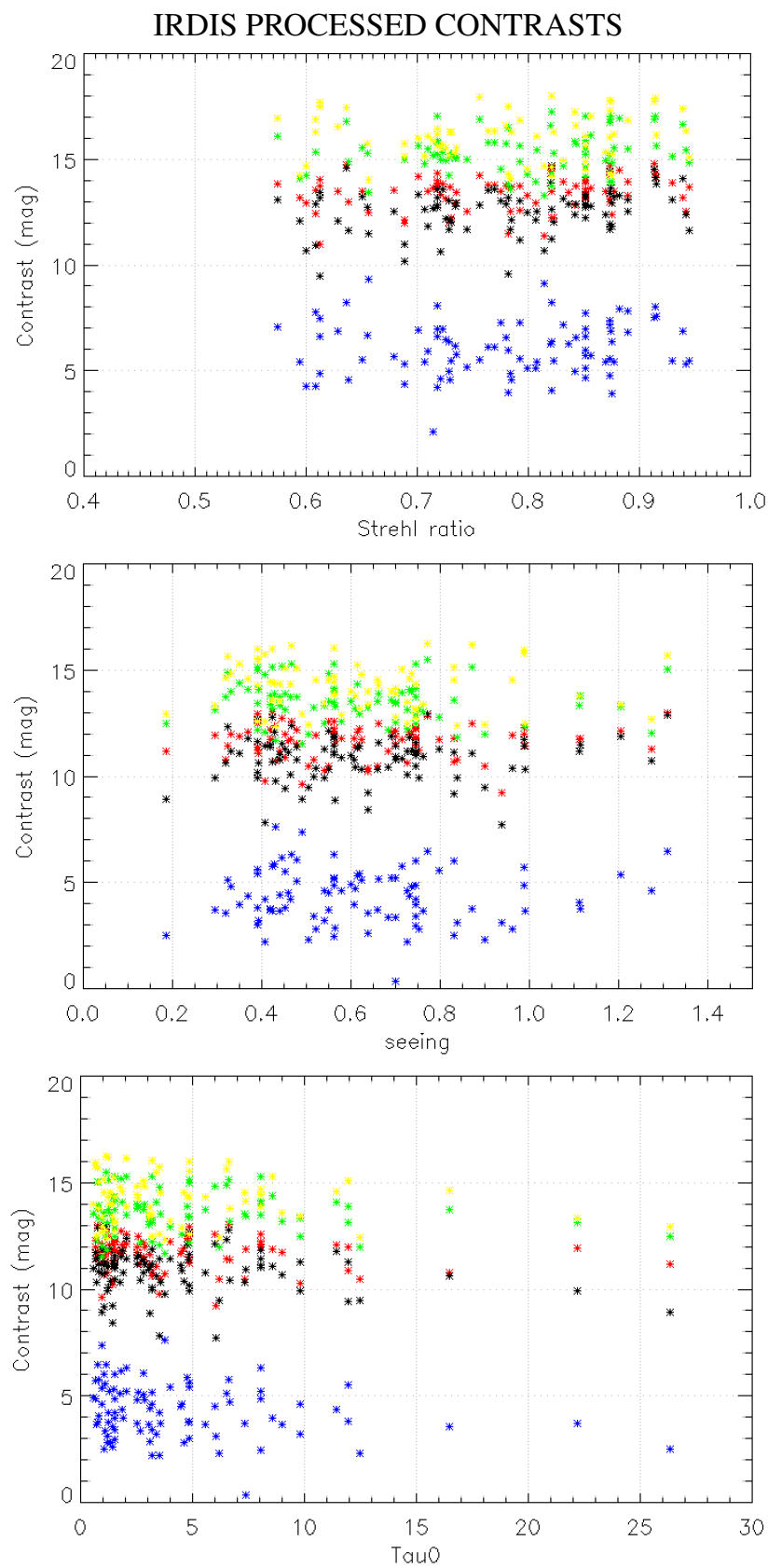

Fig. B.1. IRDIS raw (left) and processed (right) contrasts in $\mathrm{H} 2$ band at various separations, computed using TLOCI estimated as function of the observing conditions in irdifs mode. Blue is for 100 mas separation, black for 500 mas separation, red for 1000 mas separation, green for 2000 mas separation, and yellow for 4000 mas separations. The seeing is expressed in arcseconds and the $\tau_{0}$ in milliseconds. 
M. Langlois et al.: The SPHERE infrared survey for exoplanets (SHINE). II.
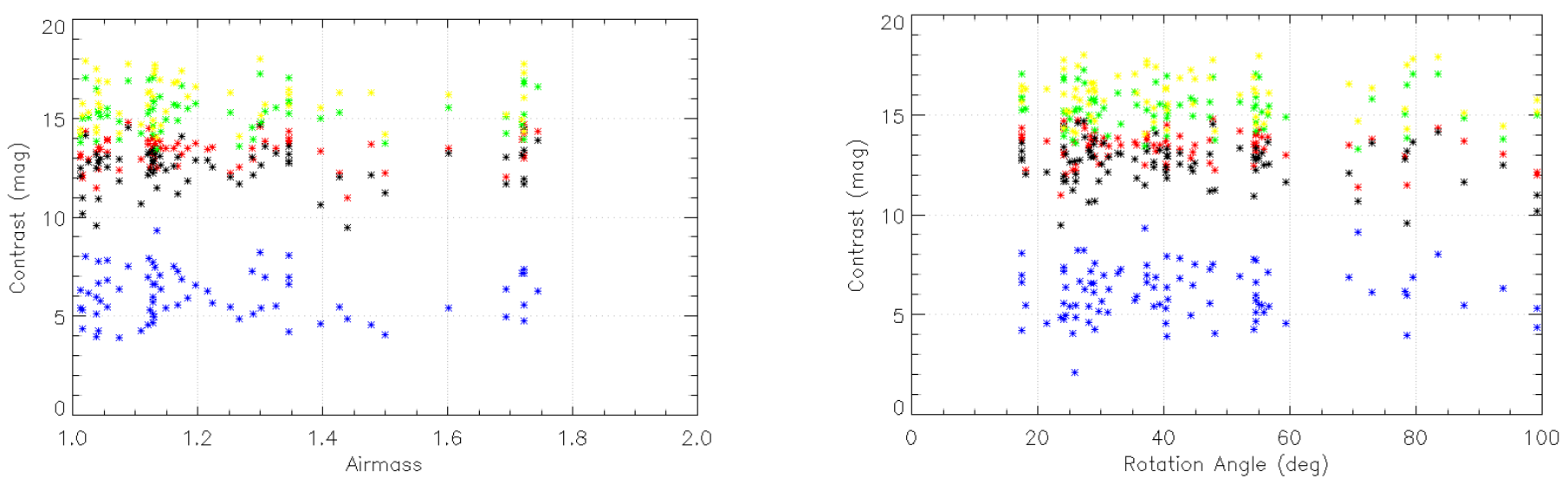

Fig. B.2. $5 \sigma$ IRDIS processed contrasts at various separations computed using TLOCI estimated as function of the observing conditions (airmass and rotation angle) in irdifs mode. Blue is for 100 mas separation, black for 500 mas separation, red for 1000 mas separation, green for 2000 mas separation, and yellow for 4000 mas separations.
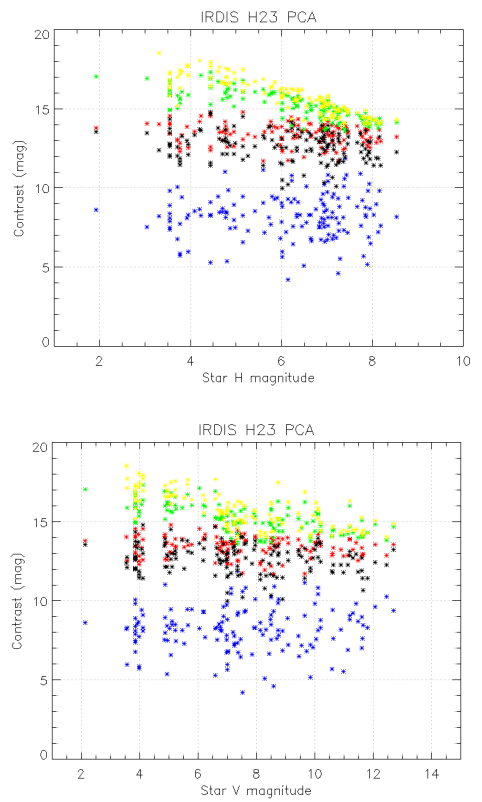
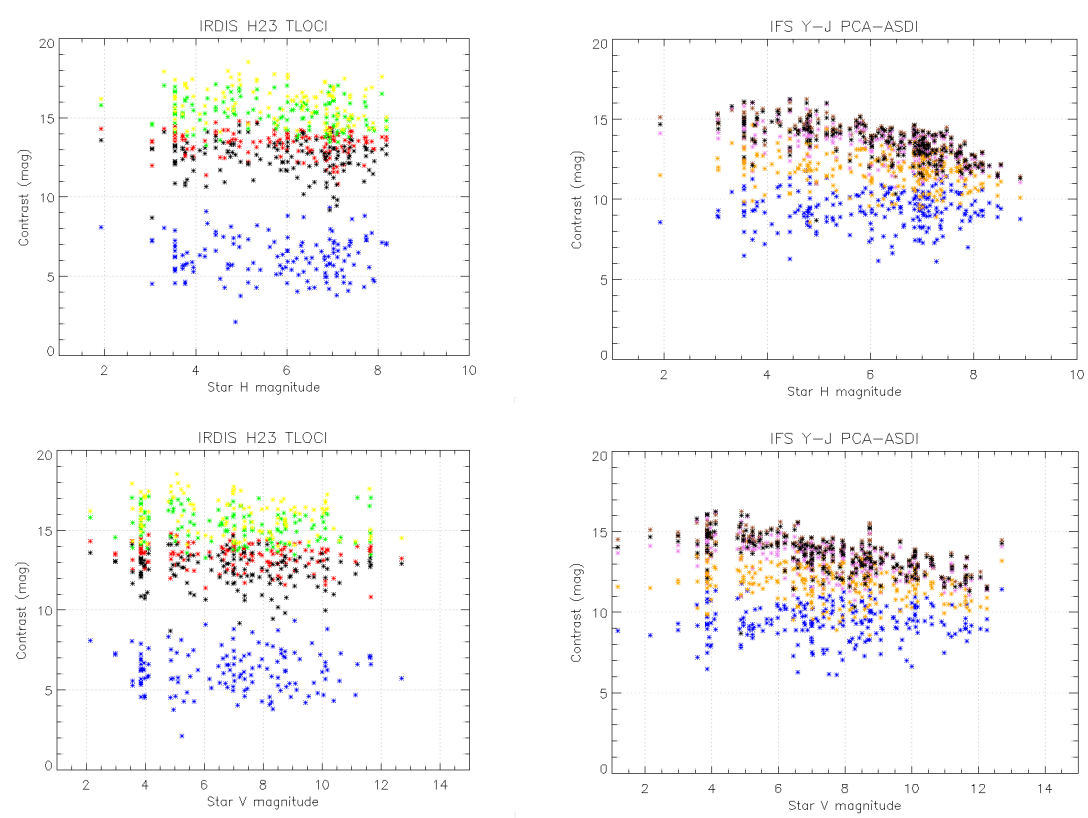

Fig. B.3. IRDIFS contrasts estimated at various separations as function of the sample stars $V$ and $H$ magnitude. Both IRDIS and IFS contrasts are shown separately. IRDIS contrasts using PCA reduction and TLOCI are shown in the left and middle figures. The color code for IRDIS is as follows: blue is for 100 mas separation, black for 500 mas separation, red for 1000 mas separation, green for 2000 mas separation, and yellow for 4000 mas separations. IFS contrasts using PCA-ASDI reduction are shown on the right side figure. The color code for IFS is as follow. Blue is for 100 mas separation, orange is for 200 mas separation, pink is for 400 mas separation, black for 500 mas separation, brown is for 700 mas separations. 
IFS RAW CONTRASTS
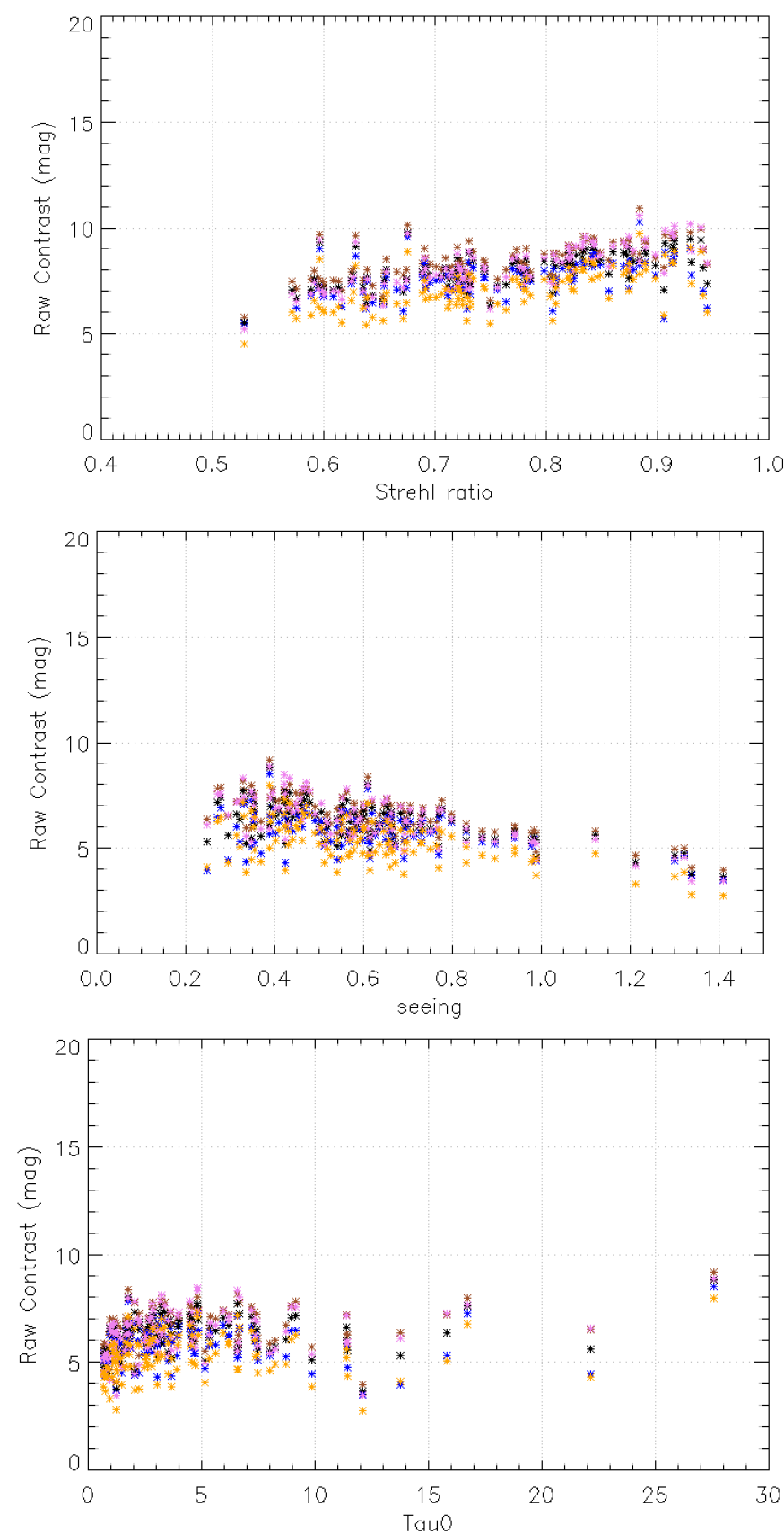

IFS PROCESSED CONTRASTS
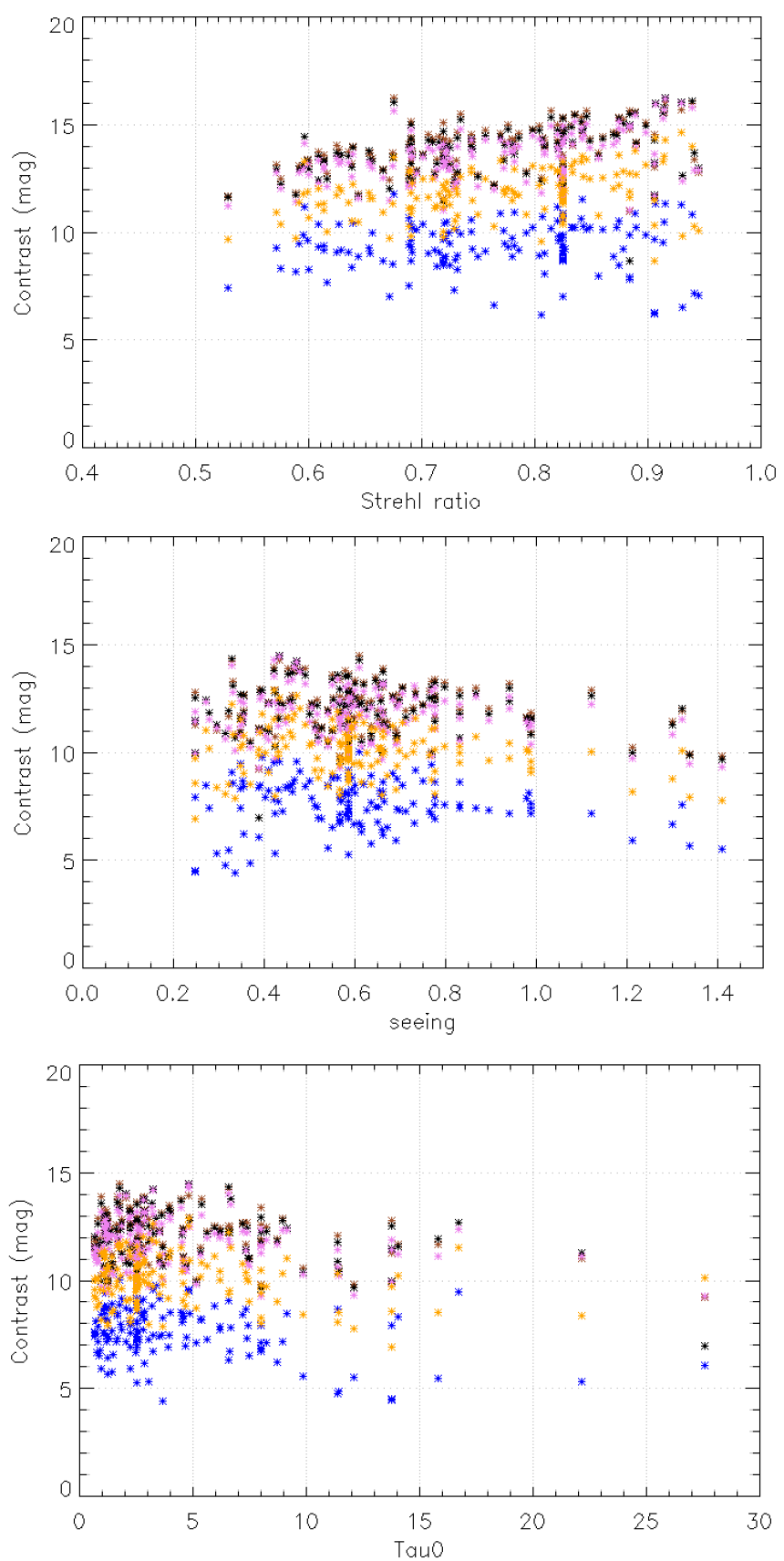

Fig. B.4. IFS raw (left) and processed (right) contrasts in $Y J$ bands at various separations computed using PCA ASDI estimated as function of the observing conditions in irdifs mode. Blue is for 100 mas separation, orange for 200 mas separation and pink for 400 mas separations, black for 500 mas separation, brown for 700 mas separation. 